ARTICLE

\title{
Dysregulation of transition metal ion homeostasis is the molecular basis for cadmium toxicity in Streptococcus pneumoniae
}

\author{
Stephanie L. Begg1, Bart A. Eijkelkamp', Zhenyao Luo 2,3,4, Rafael M. Couñago 2,3,4, Jacqueline R. Morey ${ }^{1}$, \\ Megan J. Maher ${ }^{5}$, Cheryl-lynn Y. Ong ${ }^{2,3}$, Alastair G. McEwan²,3, Bostjan Kobe 2,3,4, Megan L. O'Mara ${ }^{2,6}$, \\ James C. Paton ${ }^{1} \&$ Christopher A. McDevitt ${ }^{1}$
}

Cadmium is a transition metal ion that is highly toxic in biological systems. Although relatively rare in the Earth's crust, anthropogenic release of cadmium since industrialization has increased biogeochemical cycling and the abundance of the ion in the biosphere. Despite this, the molecular basis of its toxicity remains unclear. Here we combine metal-accumulation assays, high-resolution structural data and biochemical analyses to show that cadmium toxicity, in Streptococcus pneumoniae, occurs via perturbation of first row transition metal ion homeostasis. We show that cadmium uptake reduces the millimolar cellular accumulation of manganese and zinc, and thereby increases sensitivity to oxidative stress. Despite this, high cellular concentrations of cadmium $(\sim 17 \mathrm{mM})$ are tolerated, with negligible impact on growth or sensitivity to oxidative stress, when manganese and glutathione are abundant. Collectively, this work provides insight into the molecular basis of cadmium toxicity in prokaryotes, and the connection between cadmium accumulation and oxidative stress.

\footnotetext{
${ }^{1}$ Research Centre for Infectious Diseases, School of Biological Sciences, The University of Adelaide, Adelaide, South Australia 4072 , Australia. ${ }^{2}$ School of Chemistry and Molecular Biosciences, University of Queensland, Brisbane, Queensland 4072, Australia. ${ }^{3}$ Australian Infectious Diseases Research Centre, University of Queensland, Brisbane, Queensland 4072, Australia. ${ }^{4}$ Institute for Molecular Bioscience, University of Queensland, Brisbane, Queensland 4072, Australia. ${ }^{5}$ La Trobe Institute for Molecular Science, La Trobe University, Melbourne, Victoria 3086, Australia. ${ }^{6}$ School of Mathematics and Physics, University of Queensland, Brisbane, Queensland 4072, Australia. Correspondence and requests for materials should be addressed to C.A.M. (email: christopher.mcdevitt@adelaide.edu.au).
} 
G lobal cadmium $(\mathrm{Cd})$ production has risen by $>1,000$-fold since the beginning of the twentieth century to $\sim 20,000$ tons per year ${ }^{1}$. Correspondingly, anthropogenic release of $\mathrm{Cd}$ into the atmosphere now significantly outstrips natural fluxes and predominantly occurs via non-ferrous ore processing, combustion of fossil fuels, and manufacturing, use and disposal of Cd-containing products ${ }^{1}$. As Cd cannot be degraded, its flux into marine and terrestrial ecosystems has increased the risk of exposure as it accumulates in the food chain, and it is estimated that humans ingest $30 \mu \mathrm{g}$ of Cd every day ${ }^{1,2}$. Cd, which occurs as the divalent cation in the natural environment, is acutely toxic to all forms of life, although there are exceptions to this rule for organisms that have evolved in environments with extremely low zinc abundance ${ }^{3}$. Cd toxicity in humans and microbes is, at a cellular level, closely associated with oxidative stress, despite the inability of $\mathrm{Cd}^{2+}$ to directly produce reactive oxygen species ${ }^{4}$. As a consequence, understanding the molecular basis for how $\mathrm{Cd}^{2+}$ causes toxicity is a crucial issue.

$\mathrm{Cd}^{2}+$ accumulation in microbes and humans primarily occurs by uptake via divalent metal transporters, such as manganese $\left(\mathrm{Mn}^{2+}\right)$ transporters, although the molecular basis for this process remains unknown ${ }^{5,6}$. We investigated $\mathrm{Cd}^{2+}$ toxicity in the Gram-positive bacterium Streptococcus pneumoniae (pneumococcus) as it has only a single $\mathrm{Mn}^{2+}$-specific uptake pathway. The pneumococcus acquires $\mathrm{Mn}^{2+}$ via the PsaBCA permease, which comprises the ATP-binding cassette (ABC) transporter, $\mathrm{PsaBC}$, and a cell-surface solute-binding protein (SBP), PsaA. The Psa permease is essential for both $\mathrm{Mn}^{2+}$ uptake and in vivo virulence ${ }^{7-9}$. Similar to other $\mathrm{ABC}$ permeases, the SBP PsaA defines the specificity of the uptake pathway. However, our recent studies showed that the functional specificity of the Psa permease in $\mathrm{Mn}^{2+}$ acquisition arose not from the specificity of $\mathrm{Mn}^{2+}$ binding by PsaA, but instead resulted from the concerted action of protein metal ion coordination chemistry and structural rearrangements that prevented release of other divalent transition metal ions, such as $\mathrm{Zn}^{2+}$, into the permease, despite their ability to bind to $\mathrm{PsaA}^{8}$. Thus, although it is conceivable that $\mathrm{Cd}^{2}+$ may interact with the Psa permease, whether it causes injury to the cell by non-productively competing for metal ion uptake or by accumulation in the cytosol remains unclear.

Here we use a combination of in vitro, in vivo and structural analyses to investigate how $\mathrm{Cd}^{2}+$ impacts transition metal homeostasis in $S$. pneumoniae. We show that $S$. pneumoniae accumulates millimolar concentrations of transition metal ions, and that $\mathrm{Cd}^{2+}$ dysregulates metal ion homeostasis by perturbing the $\mathrm{Mn}^{2+}$ and $\mathrm{Zn}^{2+}$ uptake and efflux pathways, although via strikingly different mechanisms. Manganese accumulation is most affected, and this arises from $\mathrm{Cd}^{2}+$ competing with $\mathrm{Mn}^{2+}$ ions for uptake via the Psa permease and the concomitant upregulation of the $\mathrm{Mn}^{2+}$-efflux pathway. High-resolution structural analyses reveal that although the $\mathrm{Mn}^{2+}$-specific Psa permease uses coordination chemistry and structural features to prevent the mistranslocation of other ions, such as $\mathrm{Zn}^{2+}$, the larger ionic radius of $\mathrm{Cd}^{2}+$ enables this metal to circumvent these regulatory mechanisms. The increased sensitivity to oxidative stress associated with $\mathrm{Cd}^{2}+$ toxicity is due to impaired $\mathrm{Mn}^{2+}$ acquisition and not directly due to the intracellular accumulation of $\mathrm{Cd}^{2+}$ ions, which are buffered on cellular glutathione. Collectively, these findings highlight the challenge that biological systems encounter when non-physiological elements enter the biosphere. This study provides novel insights into the intracellular concentrations of transition metal ions in a Gram-positive cell, the mechanisms associated with their homeostasis, and how $\mathrm{Cd}^{2+}$ dysregulates these processes.

\section{Results}

S. pneumoniae accumulates high concentrations of metal ions. We first sought to determine the cellular quotient of first row transition metal ions in $S$. pneumoniae. Inductively coupled plasma-mass spectrometry (ICP-MS) data were combined with scanning electron microscopy analyses and colony-forming unit (c.f.u.) counts to derive the intracellular metal ion accumulation data in molar units. In this context, the intracellular metal ion concentration corresponds to the total accumulation of transition metal ions in the cytoplasmic compartment of S. pneumoniae and represents all cytoplasmic metal ions, from those bound in tight metal-protein complexes to those occurring as labile pools of exchangeable ions. Although the total number of metal ions per cell was similar to that previously reported for Escherichia coli ${ }^{10}$, the pneumococcus has a smaller mean ( \pm s.d.) cell volume, $0.113 \pm 0.04 \mathrm{fl}(n=44 ;$ Table 1$)$, and only a single cellular compartment, which results in the total cellular metal ion concentrations being in the millimolar range (Fig. 1a). The Irving-Williams stability series is the near-universal order of metal-protein-binding affinity, which arises from the imperfect steric selection of metal ions that occurs within protein-metalbinding sites. In order of increasing atomic number this can be represented as $\mathrm{Mn}^{2+}<\mathrm{Fe}^{2+}<\mathrm{Co}^{2+}<\mathrm{Ni}^{2+}<\mathrm{Cu}^{2+}>\mathrm{Zn}^{2+}$ (ref. 11). Our data also revealed that the total cellular quotient of $\mathrm{Mn}^{2}+(8.6 \pm 0.5 \mathrm{mM})$ was higher than the accumulation of other more competitive ions, such as $\mathrm{Zn}^{2+}(6.8 \pm 0.4 \mathrm{mM})$. Hence, in S. pneumoniae, the accumulation of $\mathrm{Mn}^{2+}$ to higher intracellular concentrations may be one factor that aids in increasing its bioavailability, relative to more competitive ions, such as $\mathrm{Zn}^{2+}$, and thereby aid in its acquisition by proteins that require $\mathrm{Mn}^{2+}$. We then proceeded to ascertain the impact of $\mathrm{Cd}^{2+}$ on the cellular accumulation of transition metal ions.

Cd dysregulates transition metal homeostasis. Titrations of $\mathrm{Cd}^{2+}$ into a cation-defined medium (CDM), with a constant concentration of $\mathrm{Mn}^{2}+(1 \mu \mathrm{M})$, showed that pneumococcal growth was perturbed (Fig. 1b and Supplementary Fig. 1a), but that this growth defect could essentially be abrogated by the addition of a stoichiometric concentration of $\mathrm{Mn}^{2+}$ to the $\mathrm{Cd}^{2+}$-supplemented CDM (Fig. 1c and Supplementary Fig. 1b). $S$. pneumoniae growth in the presence of $30 \mu \mathrm{M} \mathrm{Cd}^{2}+$ reduced the cellular concentrations of $\mathrm{Mn}^{2+}$ and $\mathrm{Zn}^{2+}$. Manganese accumulation was most affected, with a reduction of $\sim 90 \%$ (Fig. 1a; $1 \mu \mathrm{M} \mathrm{Mn}^{2+}: 30 \mu \mathrm{M} \mathrm{Cd}^{2+}, P<0.0001$ (one-way analysis of variance (ANOVA))), despite no significant effect on cell volume (Table 1; $1 \mu \mathrm{M} \mathrm{Mn}^{2+}: 30 \mu \mathrm{M} \mathrm{Cd}^{2+}, P=0.376$ (one-way ANOVA)). Supplementation with $\mathrm{Mn}^{2+}$ restored pneumococcal $\mathrm{Mn}^{2+}$ accumulation to $\sim 60 \%$ of the unchallenged levels (Fig. 1a

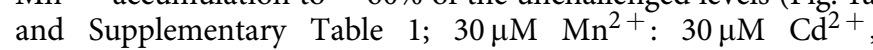
$P<0.0001$ (one-way ANOVA)). Zinc also showed a significant decrease in accumulation in the presence of $\mathrm{Cd}^{2}+$ (Fig. 1a; $1 \mu \mathrm{M}$ $\mathrm{Mn}^{2+}: 30 \mu \mathrm{M} \mathrm{Cd}{ }^{2+}, P<0.0001 ; 30 \mu \mathrm{M} \mathrm{Mn}^{2+}: 30 \mu \mathrm{M} \mathrm{Cd}^{2+}$, $P<0.0001$ (one-way ANOVA)). $\mathrm{Cd}^{2}+$ accumulation increased in the presence of $30 \mu \mathrm{M} \mathrm{Cd}^{2+}$, with or without $\mathrm{Mn}^{2+}$ (Fig. 1a). The degree to which $\mathrm{Cd}^{2+}$ was accumulated was more than that

Table 1 | S. pneumoniae cell volume parameters.
Strain

D39 $1 \mu \mathrm{M} \mathrm{Mn}{ }^{2+}$

D39 $1 \mu \mathrm{M} \mathrm{Mn}^{2+}: 30 \mu \mathrm{M} \mathrm{Cd} d^{2+}$ D39 $30 \mu \mathrm{M} \mathrm{Mn}^{2+}: 30 \mu \mathrm{M} \mathrm{Cd}^{2+}$
Cell volume (fl)*

$0.113 \pm 0.04$

$0.124 \pm 0.07$

$0.127 \pm 0.07$
${ }^{\star}$ The data correspond to the mean \pm s.d. from $>40$ independent measurements. 
a

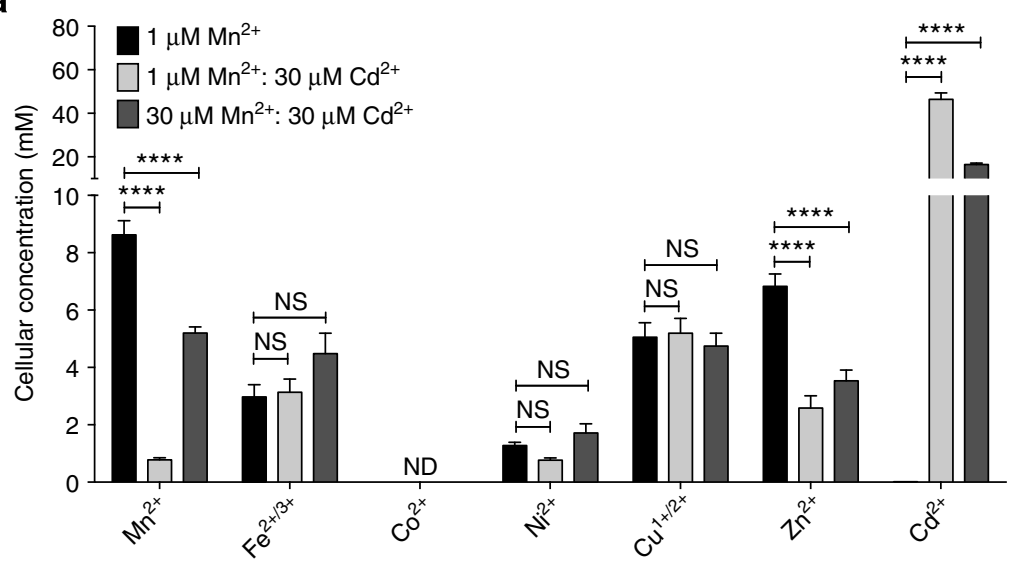

b

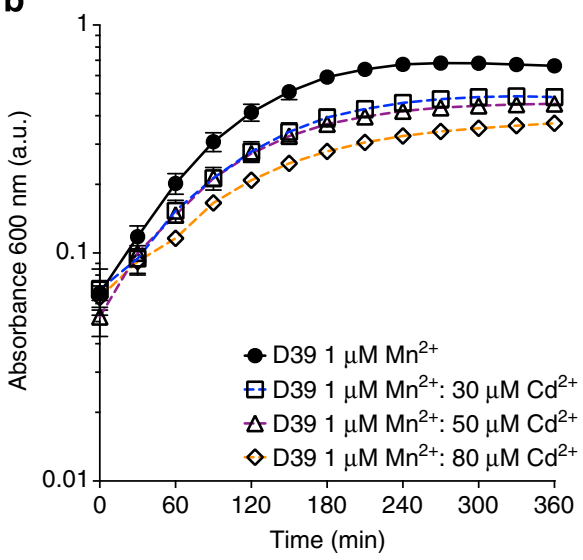

c

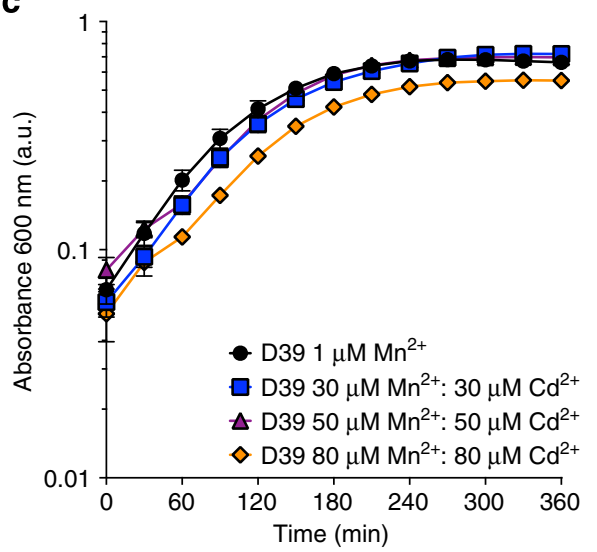

d

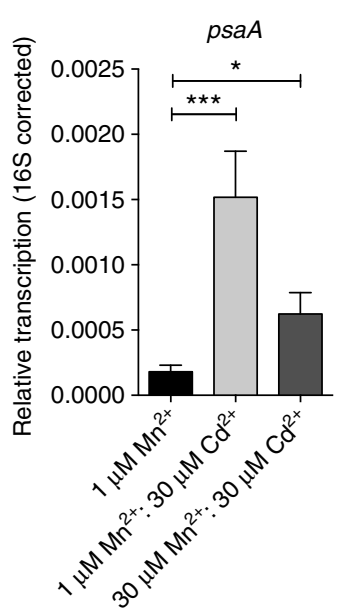

e

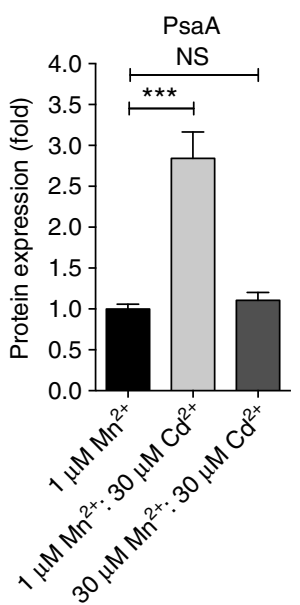

f

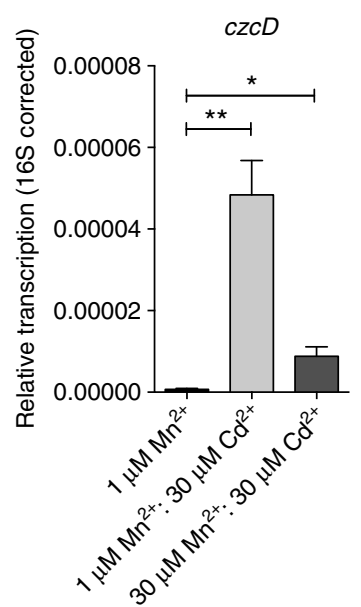

Figure 1. | Impact of $\mathbf{C d}^{\mathbf{2}+}$ on S. pneumoniae growth and first row transition metal homeostasis. (a) Total cellular metal ion accumulation of S. pneumoniae represented as the mean ( \pm s.e.m.) concentration of ions per cell (determined as c.f.u.) of known cell volume (determined by scanning electron microscopy (SEM)) grown in the specified conditions. The values represent the total cellular metal ion quota, that is, bound, buffered and unbound metal ions, and are from at least three independent biological experiments. Metal ions assessed were $\mathrm{Mn}^{2+}, \mathrm{Fe}^{2+/ 3+}, \mathrm{Co}^{2+}, \mathrm{Ni}^{2+}, \mathrm{Cu}^{1+/ 2+}, \mathrm{Zn}^{2+}$ and $\mathrm{Cd}^{2+}$. The statistical significance of the differences in the mean metal concentrations was determined by a one-way ANOVA (ND, not detected; NS, not significant and $\left.{ }^{\star \star \star \star} P<0.0001\right)$. (b,c) S. pneumoniae (D39) grown in CDM supplemented with metal ions as indicated. The data correspond to mean ( \pm s.e.m.) absorbance at $600 \mathrm{~nm}$ measurements from three independent biological experiments. Errors bars, where not visible, are overlapped by the representative symbols. (d) Relative transcription, corrected to $16 \mathrm{~S}$ rRNA, of psaA by S. pneumoniae when grown in CDM supplemented with $1 \mu \mathrm{M}$ Mn ${ }^{2+}$ (black), $1 \mu \mathrm{M} \mathrm{Mn}^{2+}: 30 \mu \mathrm{M} \mathrm{Cd}{ }^{2+}$ (light grey) and $30 \mu \mathrm{M} \mathrm{Mn}^{2+}: 30 \mu \mathrm{M} \mathrm{Cd}^{2+}$ (dark grey). (e) Quantitative immunoblot data of PsaA (Supplementary Fig. 2), from equivalent total protein loadings of whole-cell extracts, from S. pneumoniae when grown in CDM supplemented with $1 \mu M M^{2}+$ (black), $1 \mu M$ $\mathrm{Mn}^{2+}: 30 \mu \mathrm{M} \mathrm{Cd}^{2+}$ (light grey) and $30 \mu \mathrm{M} \mathrm{Mn}^{2+}: 30 \mu \mathrm{M} \mathrm{Cd}^{2+}$ (dark grey). (f) Relative transcription, corrected to 16S rRNA, of czcD by S. pneumoniae when grown in CDM supplemented with $1 \mu \mathrm{M} \mathrm{Mn}^{2+}$ (black), $1 \mu \mathrm{M} \mathrm{Mn}^{2+}: 30 \mu \mathrm{M} \mathrm{Cd}^{2+}$ (light grey) and $30 \mu \mathrm{M} \mathrm{Mn} \mathrm{M}^{2+}: 30 \mu \mathrm{M} \mathrm{Cd^{2+ }}$ (dark grey). The data correspond to the mean ( \pm s.e.m.) of three independent biological experiments. The statistical significance of the differences in the mean data was determined by two-tailed unpaired $t$-tests $\left({ }^{\star} P<0.05,{ }^{\star \star} P<0.01\right.$ and $\left.{ }^{\star \star \star} P<0.001\right)$.

of any other transition metal ion in the pneumococcus, despite having no physiological role. At $1 \mu \mathrm{M} \mathrm{Mn}^{2+}: 30 \mu \mathrm{M} \mathrm{Cd}^{2+}$ in the growth medium, intracellular $\mathrm{Cd}^{2}+$ concentration was $46.4 \pm 2.9 \mathrm{mM}$. $\mathrm{Cd}^{2}+$ accumulation was reduced by nearly $70 \%$ on the addition of $30 \mu \mathrm{M} \mathrm{Mn} \mathrm{Mn}^{2}$ to the growth medium $\left(16.5 \pm 0.7 \mathrm{mM} \mathrm{Cd}^{2+}\right)$, but cellular accumulation of the ion still remained more than that of the other first row transition metal ions.

We then analysed the impact of $\mathrm{Cd}^{2+}$ on transition metal ion homeostatic pathways. Transcriptional analyses revealed that psaA transcription increased by 8.4 -fold $(P=0.0007$ (two-tailed unpaired $t$-test) $)$ and $\mathrm{PsaA}$ expression by $2.8 \pm 0.3$-fold $(P<0.0001$ (two-tailed unpaired $t$-test) $)$ when grown in the presence of $30 \mu \mathrm{M} \mathrm{Cd}^{2+}$ (Fig. 1d,e), consistent with the $\mathrm{Mn}^{2+}$. responsive transcriptional regulator, PsaR, sensing the $\mathrm{Mn}^{2+}$ depletion ${ }^{7,12}$. Transcription of $p s a A$ remained elevated during growth supplemented with $30 \mu \mathrm{M} \mathrm{Mn}{ }^{2}+$ albeit to a lesser, but still significant, extent (3.5-fold, $P=0.0135$ (two-tailed unpaired $t$-test)), although PsaA expression did not appear to be significantly increased (Fig. 1d,e). Zinc accumulation in $S$. pneumoniae also occurs via an $\mathrm{ABC}$ permease, the Adc permease $e^{9,13,14}$. Transcriptional analysis of the $\mathrm{Zn}^{2+}$-responsive regulator, $a d c R^{15}$, and the $\mathrm{Zn}^{2+}$-recruiting SBPs associated with the Adc permease, $a d c A$ and adcAII (refs 13,14,16), did not show significant changes in the presence of $\mathrm{Cd}^{2+}$ (Supplementary Fig. 3). Despite this, the $\mathrm{Zn}^{2}+$ accumulation levels $(2.6 \pm 0.4 \mathrm{mM})$ were comparable to strains without a functional Adc permease, $\triangle a d c C B A(2.7 \pm 0.3 \mathrm{mM})$ and $\triangle a d c A / \triangle a d c A I I(2.5 \pm 0.3 \mathrm{mM})$. 
The perturbation of $\mathrm{Zn}^{2+}$ acquisition was not due to competition for the Adc permease as the dominant $\mathrm{Zn}^{2+}$-recruiting SBP, AdcA, showed very poor interaction with $\mathrm{Cd}^{2+}$ by comparison with $\mathrm{Zn}^{2}+$ (Supplementary Fig. 3). Taken together, these findings implicate $\mathrm{Cd}^{2+}$ as being able to exert an effect on the $\mathrm{Zn}^{2+}$ responsive transcriptional regulators. S. pneumoniae uses two $\mathrm{Zn}^{2+}$-responsive transcriptional regulators: AdcR, which regulates the $\mathrm{Zn}^{2+}$-uptake machinery described above, and SczA, which regulates transcription of the cation diffusion facilitator efflux protein $\mathrm{CzcD}^{15,17}$. Consequently, we examined whether $\mathrm{CzcD}$ was also affected by $\mathrm{Cd}^{2}+$ abundance. Growth of S. pneumoniae in CDM with $1 \mu \mathrm{M} \mathrm{Mn} \mathrm{Mn}^{2+}: 30 \mu \mathrm{M} \mathrm{\textrm {Cd } ^ { 2 + }}$ increased $c z c D$ transcription by $\sim 70$-fold $(P=0.0011$ (twotailed unpaired $t$-test); Fig. 1f), while supplementation with $\mathrm{Mn}^{2+}\left(30 \mu \mathrm{M} \mathrm{Mn}^{2+}: 30 \mu \mathrm{M} \mathrm{Cd}^{2+}\right)$ reduced this to only $\sim 12.7-$ fold more than cells grown in unsupplemented CDM (Fig. 1f). Although CzcD from Cupriavidus metallidurans is protective against $\mathrm{Co}^{2+}, \mathrm{Zn}^{2+}$ and $\mathrm{Cd}^{2+}$ ions ${ }^{18}$, there is no evidence to suggest that the $S$. pneumoniae orthologue has similar polyspecificity. Consequently, we examined an isogenic $\Delta c z c D$ mutant strain to ascertain whether pneumococcal $c z c D$ contributed to $\mathrm{Cd}^{2+}$ resistance. The $S$. pneumoniae $\Delta c z c D$ strain was hypersensitive to extracellular $\mathrm{Zn}^{2+}$, consistent with a role in $\mathrm{Zn}^{2+}$ homeostasis (Fig. 2). Furthermore, the $\mathrm{Cd}^{2+}$-induced efflux of $\mathrm{Zn}^{2}+$ observed in wild-type $S$. pneumoniae was abolished in the

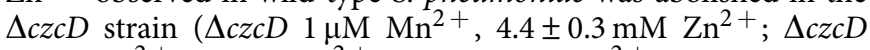
$1 \mu \mathrm{M} \mathrm{Mn}{ }^{2+}: 30 \mu \mathrm{M} \mathrm{Cd}{ }^{2+} 4.2 \pm 0.3 \mathrm{mM} \mathrm{Zn}^{2+} ; P=0.78$ (twotailed unpaired $t$-test)). However, although $\mathrm{Cd}^{2+}$ accumulation in the $\Delta c z c D$ strain showed a mild elevation of $\sim 1.4$-fold compared with the wild-type, the growth phenotypes of the $\Delta c z c D$ strain were indistinguishable from the wild-type strain and also showed no increase in susceptibility to extracellular $\mathrm{Cd}^{2+}$ (Fig. 2 and Supplementary Fig. 4). Taken together, these data indicate that, despite the upregulation of $S$. pneumoniae $c z c D$ in response to $\mathrm{Cd}^{2+}$ exposure, $\mathrm{CzcD}$ is primarily a $\mathrm{Zn}^{2}+$ efflux transporter and appears to serve, at best, only a minor protective role against $\mathrm{Cd}^{2+}$.

In addition to $c z c D$, the pneumococcal genome encodes four other putative metal ion efflux pathways. These are as follows: spd1384 (mntE), a paralogue of CzcD involved in $\mathrm{Mn}^{2+}$ efflux ${ }^{19}$; spd1438 (cadD), a membrane protein of unknown function associated with $\mathrm{Cd}^{2}+$ tolerance ${ }^{20}$; two P-type ATPases, spd0635 $(\operatorname{cop} A)$, which is responsible for $\mathrm{Cu}^{1+}$ efflux ${ }^{21}$; and spd1927, which has homology to $p m t A$, a PerR-regulated gene in S. pyogenes associated with $\mathrm{Zn}^{2+}$ resistance ${ }^{22}$. The mntE gene has previously been reported to be constitutively expressed and not responsive to cellular $\mathrm{Mn}^{2+}$ abundance ${ }^{19}$. Here, we observed that extracellular $\mathrm{Cd}^{2+}$ induced a 2.1 -fold increase in $m n t E$ transcription $(P=0.0211$ (two-tailed unpaired $t$-test); Supplementary Fig. 5a). On supplementation with $30 \mu \mathrm{M}$ $\mathrm{Mn}^{2}+$, mntE transcription was reduced to non-challenged levels $(P=0.3665$ (two-tailed unpaired $t$-test) Supplementary Fig. 5a), suggesting that intracellular $\mathrm{Cd}^{2}+$ concentrations modulated $m n t E$ expression via an unknown metal-responsive regulator. We then analysed an isogenic $\Delta m n t E$ strain, which lacks the primary $\mathrm{Mn}^{2+}$-efflux pathway ${ }^{19}$, for sensitivity to $\mathrm{Cd}^{2}+$. Consistent with $\mathrm{Cd}^{2}+$ also perturbing $\mathrm{Mn}^{2}+$ homeostasis via MntE, the $\Delta m n t E$ strain retained a higher cellular $\mathrm{Mn}^{2}$ concentration $(2.53 \pm 0.1 \mathrm{mM})$ than the wild-type $(0.78 \pm 0.07 \mathrm{mM})(P<0.0001$ (two-tailed unpaired $t$-test) $)$, when

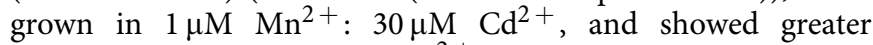
resistance to extracellular $\mathrm{Cd}^{2+}$ (Fig. 2). The cadD gene showed a similar trend to $c z c D$ and $m n t E$ with a $\sim 3.6$-fold increase in transcription when challenged with $30 \mu \mathrm{M} \mathrm{Cd} \mathrm{Cd}^{2+}$ $(P<0.0001$ (two-tailed unpaired $t$-test); Supplementary Fig. $5 b)$, followed by abrogation when supplemented with $30 \mu \mathrm{M} \mathrm{Mn}{ }^{2+}$.
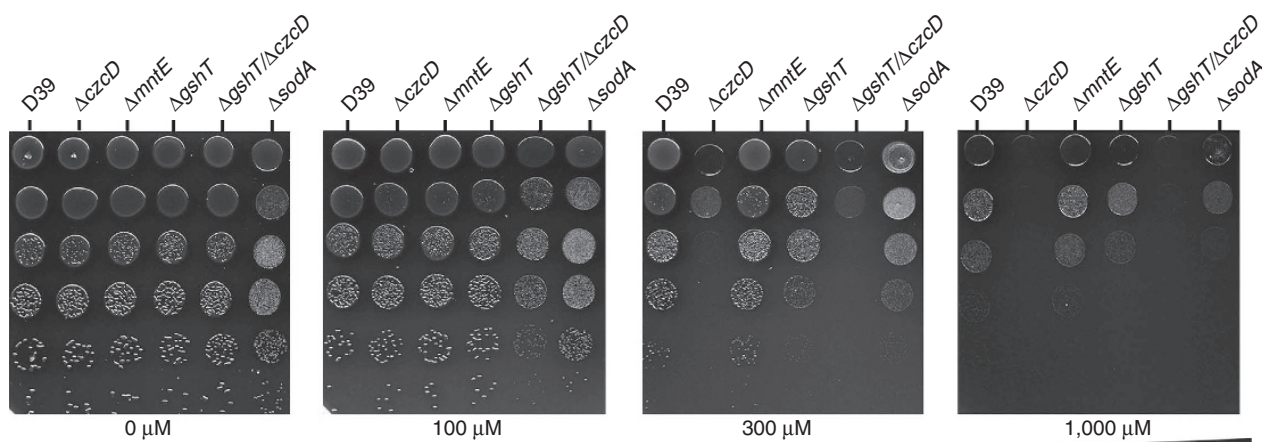

$\mathrm{Zn}^{2+}$
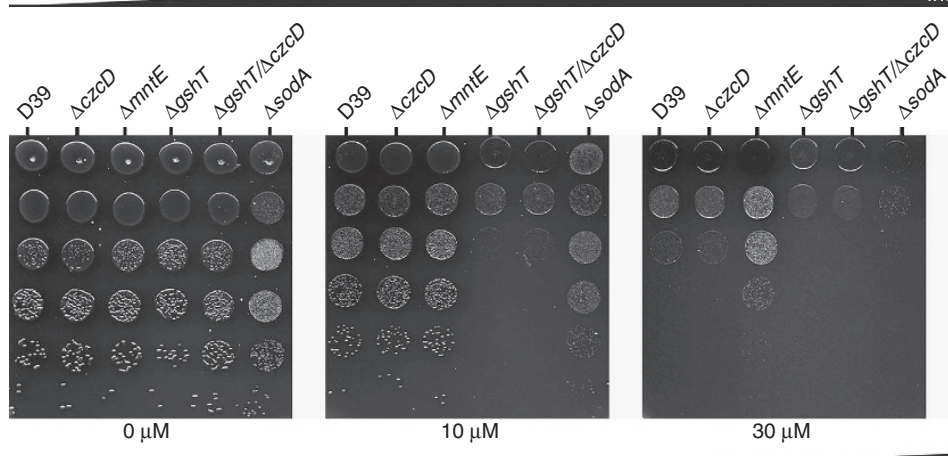

Increasing metal-ion concentration

$\mathrm{Cd}^{2+}$

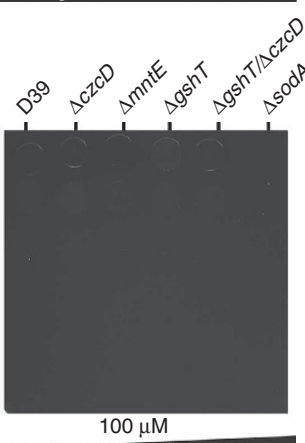

Increasing metal-ion concentration

Figure 2 | Phenotypic impact of metal ions on S. pneumoniae growth. Drop test analysis of S. pneumoniae wild-type (D39), $\Delta c z c D, \Delta m n t E, \Delta g s h T$, $\Delta g s h T / \Delta c z c D$ and $\Delta$ sodA on blood agar (BA) supplemented with: 0, 100, 300 and 1,000 $\mu \mathrm{M} \mathrm{ZnSO}_{4}$ or 0, 10, 30 and $100 \mu \mathrm{M} \mathrm{CdCl} 2$. Cells were grown and adjusted to $A_{600}$ of 0.4 and were serially diluted. Drops $(5 \mu \mathrm{l})$ were spotted onto the BA plate, starting from the $10^{\circ}$ dilution (top) down to the $10^{-5}$ dilution (bottom). 
However, the P-type ATPases, copA and spd1927, were transcriptionally unresponsive to $\mathrm{Cd}^{2}+$ (Supplementary Fig. $5 c, d$ ), suggesting that they were unlikely to contribute to $\mathrm{Cd}^{2}+$ management. Collectively, these data show that $\mathrm{Cd}^{2+}$ accumulation was associated with transcriptional activation of several metal ion membrane protein transporters. However, of those pathways with known efflux function, that is, $\mathrm{CzcD}$ and $\mathrm{MntE}, \mathrm{Cd}^{2+}$ ions subverted their primary functional roles and, instead, further dysregulated transition metal ion homeostasis in S. pneumoniae via amplification of $\mathrm{Zn}^{2+}$ and $\mathrm{Mn}^{2+}$ efflux, respectively. The mechanism(s) of $\mathrm{Cd}^{2}+$ efflux in $S$. pneumoniae, if any, remains to be identified.

$\mathrm{Cd}^{2+}$ competes with $\mathrm{Mn}^{2+}$ for the Psa permease. To elucidate the hitherto unexplained molecular basis for $\mathrm{Cd}^{2}+$ uptake via $\mathrm{Mn}^{2+}$ transporters, we examined the interaction of $\mathrm{Cd}^{2+}$ with the $\mathrm{Mn}^{2+}$-recruiting SBP PsaA. Although the physiological role of PsaA is in recruiting $\mathrm{Mn}^{2+}$, the metal-binding site of the protein has previously been shown to interact with other divalent transition metal ions ${ }^{8}$. However, coordination chemistry at the metal-binding site and structural dynamics prevent release of these other ions, and thereby maintain the functional role of the Psa permease in $\mathrm{Mn}^{2+}$ uptake ${ }^{8}$. Here the interaction of $\mathrm{Cd}^{2+}$ with PsaA was analysed by determining the structure of wild-type PsaA with $\mathrm{Cd}^{2+}$ at $2.0 \AA$ resolution (Fig. $3 \mathrm{a}$ and Supplementary Fig. 6). The PsaA-Cd ${ }^{2+}$ complex revealed that $\mathrm{Cd}^{2+}$ was coordinated by the metal-binding site residues His67, His139, Glu205 and Asp280 in a structure that was highly similar (rootmean-square deviation (RMSD) $<0.3 \AA$ over $242 \mathrm{C} \alpha$ atoms) to our previously reported $\mathrm{Mn}^{2+}$ - and $\mathrm{Zn}^{2+}$-bound structures ${ }^{23,24}$. Nevertheless, small re-orientations of the side chains of the metalcoordinating residues allowed the bulkier $\mathrm{Cd}^{2}+$ ion (ionic radius of $95 \mathrm{pm}$ for $\mathrm{Cd}^{2+}$ versus $84 \mathrm{pm}$ for $\mathrm{Mn}^{2+}$ ) to be accommodated into the protein-metal-binding site (Fig. $3 \mathrm{~b}$ and Supplementary Table 2). In the PsaA-Cd ${ }^{2}$ structure, the distances between metal-coordinating atoms and the metal ion were increased by $\sim 0.2 \AA$ compared with those for PsaA- $\mathrm{Zn}^{2}+$ (Supplementary Table 2). Similar increases in metal-protein distances have been observed for the $\mathrm{Cd}^{2+}$-thiolate-binding sites of $\mathrm{Cd}^{2+}$. a

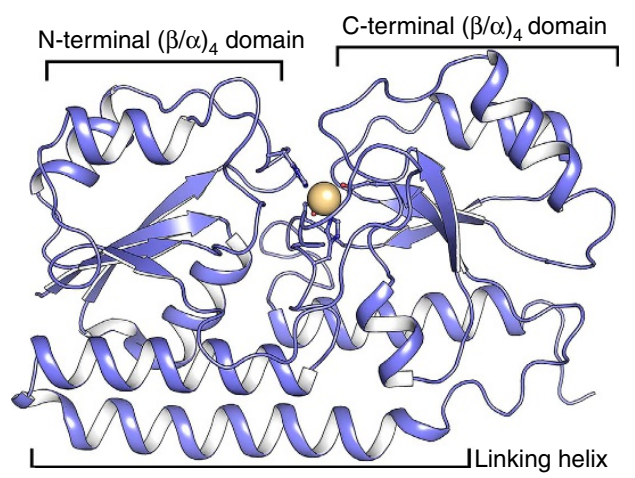

b

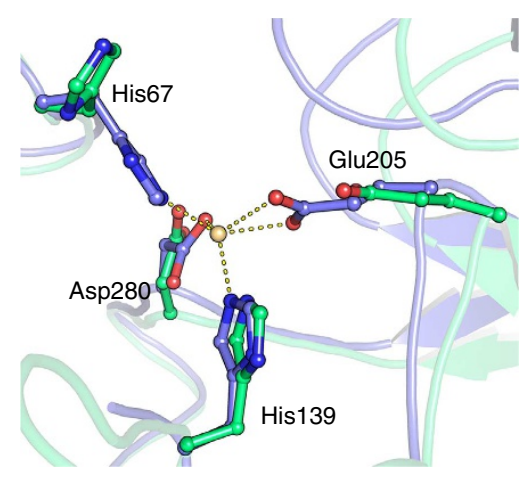

d

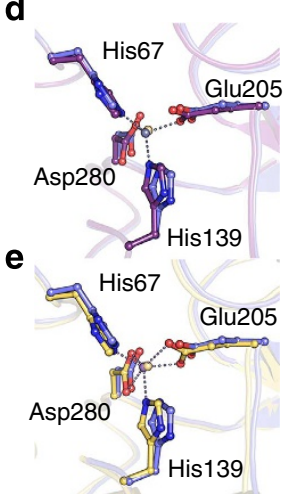

C

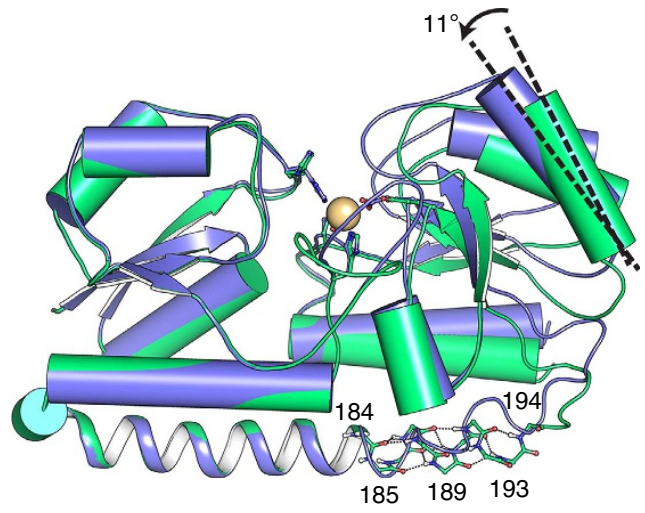

$\mathbf{f}$

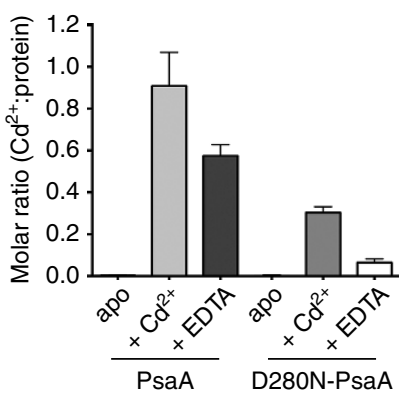

Figure 3 | Structure of $\mathbf{C d}^{\mathbf{2}+}$-bound PsaA. (a) Cartoon illustration of $\mathrm{Cd}^{2+}$-bound wild-type PsaA in closed conformation (PDB accession code 4UTP). The structure consists of $\mathrm{N}$-terminal and $\mathrm{C}$-terminal $(\beta / \alpha)_{4}$ domains with a domain-linking helix. The metal-binding site is situated between the two domains, in which a $\mathrm{Cd}^{2}+$ ion (pale-yellow sphere) is bound. (b) The metal-binding site of wild-type PsaA in the presence (light blue, PDB accession code 4UTP) and absence (green, PDB accession code 3ZK7) of $\mathrm{Cd}^{2}+$ (pale-yellow sphere), with the metal coordination shown as dashed lines. (c) Superposition of $\mathrm{Cd}^{2+}$-bound (light blue, PDB accession code 4UTP) and apo (green, PDB accession code 3ZK7) wild-type PsaA structures (C $\alpha$ atoms, residues 32-163, were used for the superposition). The linking helix is shown in cartoon representation, with other helices shown as cylinders. The crossing angle of the $C$-terminal $(\beta / \alpha)_{4}$ domains between the structures is marked. The bound $C d^{2+}$ ion is shown as a pale-yellow sphere. In the apo structure, backbone atoms in the flexible region of the linking helix (residues 184-194) are shown in stick representation, with hydrogen bonds between backbone atoms as dashed lines. (d) Comparison of the metal-binding sites between $\mathrm{Cd}^{2+}$-bound (light blue, PDB accession code 4 UTP) and $\mathrm{Zn}^{2+}$-bound (purple, PDB accession code 1PSZ) wild-type PsaA structures. The metal ions are shown as spheres $\left(\mathrm{Cd}^{2+}\right.$, pale yellow; $\mathrm{Zn}^{2+}$, grey) and their coordination as dashed lines. (e) Comparison of the metal-binding sites between $\mathrm{Cd}^{2}+$-bound (light blue, PDB accession code 4UTP) and $\mathrm{Mn}^{2+}$-bound (yellow, PDB accession code 3ZTT) wild-type PsaA structures. The metal ions are shown as spheres $\left(\mathrm{Cd}^{2+}\right.$, pale yellow; $\mathrm{Mn}^{2+}$, blue) and their coordination as dashed lines. In a-e, the metal-coordinating residues are shown in stick representation. (f) In vitro metal binding of $\mathrm{Cd}^{2}+$ to wild-type or D280N variant apo-PsaA analysed by ICP-MS. Data correspond to the mean ( \pm s.d.) molar ratio of $\mathrm{Cd}^{2+}$ to the PsaA isoform for apo-PsaA (black), Cd ${ }^{2+}-$ PsaA (light grey), EDTA-Cd ${ }^{2+}-$ PsaA (dark grey). apo-D280N-PsaA (black), Cd ${ }^{2+}-\mathrm{D} 280 \mathrm{~N}-\mathrm{PsaA}$ (grey) and EDTA-Cd ${ }^{2+}-\mathrm{D} 280 \mathrm{~N}-\mathrm{PsaA}$ (white). 
substituted metallothionein and rubredoxin proteins, compared with the $\mathrm{Zn}^{2+}$-bound equivalents ${ }^{25,26}$. Although $\mathrm{Cd}^{2+}$ and $\mathrm{Zn}^{2+}$ often display similar coordination spheres in proteins, the longer bond distances observed for $\mathrm{Cd}^{2+}$ usually result in an increase in the coordination number. Accordingly, the coordination geometry in the $\mathrm{PsaA}-\mathrm{Cd}^{2}+$ structure is an intermediate between a square-pyramid and trigonal-bipyramid $(n=5)$, in contrast to the 4- and 6-coordinate sites observed for the $\mathrm{Zn}^{2+}$ - and $\mathrm{Mn}^{2+}$-bound PsaA structures, respectively (Fig. 3d,e and Supplementary Table 3). A comparison of the observed coordination of $\mathrm{PsaA}-\mathrm{Cd}^{2+}$ with other metal-binding sites of metalloprotein structures in the PDB, composed of mixed $\mathrm{N} / \mathrm{O}$ ligating atoms, revealed that $\mathrm{Cd}^{2}+$ is found in a variety of coordination geometries with coordination numbers ranging from 5 to 7 (Supplementary Table 4). Nevertheless, the only bona fide protein-metal-binding site for $\mathrm{Cd}^{2+}$ is in carbonic anhydrase from Thalassiosira weissflogii, which has been shown to be a cambialistic enzyme with $\mathrm{Zn}^{2}+$ in a tetrahedral geometry and $\mathrm{Cd}^{2+}$ in a trigonal-bipyramidal geometry ${ }^{3,27,28}$. Thus, the coordination geometry found in $\mathrm{PsaA}-\mathrm{Cd}^{2}+$ conforms to what has been observed for the only known naturally occurring $\mathrm{Cd}^{2+}$. binding protein, and for other protein structures deposited in the PDB. We further examined the interaction of metal-free (apo) PsaA with $\mathrm{Cd}^{2+}$ and observed that apo-PsaA bound $\mathrm{Cd}^{2+}$ in a 1:1 molar ratio (Fig. 3f), with a $K_{\mathrm{D}}$ of $5.6 \pm 1 \mathrm{nM}$ (Supplementary Fig. 7). As the cognate ligand, $\mathrm{Mn}^{2+}$, has a $K_{\mathrm{D}}$ of $3.3 \pm 1 \mathrm{nM}$ for PsaA, the relatively small (approximately twofold) difference in the $K_{\mathrm{D}} \mathrm{s}$ is consistent not only with the competitive effect on $\mathrm{Mn}^{2+}$ acquisition but also the continued, albeit reduced, $\mathrm{Cd}^{2+}$ accumulation during growth in the presence of equimolar

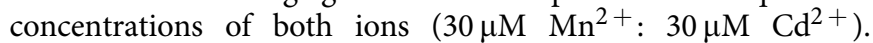
Collectively, these findings are consistent with the observed impact of $\mathrm{Cd}^{2+}$ on $\mathrm{Mn}^{2+}$ accumulation in the wild-type and mutant $S$. pneumoniae strains and indicate that it is a competitive effect mediated via PsaA. However, they also indicate that interaction of $\mathrm{Cd}^{2+}$ with PsaA is distinct from the previously characterized $\mathrm{Mn}^{2}+$ and $\mathrm{Zn}^{2}+$ interactions.

PsaA is permissive for $\mathrm{Cd}^{2+}$ binding and release. PsaA uses a 'spring-hammer'-like mechanism for metal ion binding, in which the dynamics of the metal ion-loaded protein are dictated by a combination of metal ion geometry and the distortion of a helix linking the N- and C-terminal lobes of the protein ${ }^{8}$. We further analysed the metal-binding site coordination geometry by use of an Asp280 variant isoform (PsaA-D280N), as it would be incapable of facilitating the trigonal-bipyramidal coordination observed in wild-type PsaA ${ }^{8}$. The structure of PsaA-D280N bound to $\mathrm{Cd}^{2+}$, refined to $1.7 \AA$ resolution, revealed a partially solvated $\mathrm{Cd}$ ion loosely interacting with residue Glu205 (Fig. 4a,b). The C-terminal lobe of PsaA showed minor movement $\left(\sim 1.0^{\circ}\right.$ relative to the open, metal-free conformation), but the inability of $\mathrm{Cd}^{2}+$ to ligate to residue Asp280 prevented further conformational changes. Consistent with these observations, PsaA-D280N was greatly impaired in $\mathrm{Cd}^{2+}$ binding (Fig. 3f).

We then assessed the distortion of the linking helix by monitoring the number of disrupted backbone hydrogen bonds in its flexible region (residues 184-194) in wild-type PsaA-Cd ${ }^{2+}$ (Figs $3 \mathrm{c}$ and $4 \mathrm{a}$, and Supplementary Table 5$)^{8}$. Both the hingebending angle between the $\mathrm{N}$ - and $\mathrm{C}$-terminal lobes and the number of main-chain hydrogen bonds disrupted in the residue range 184-194 corresponded more closely to the $\mathrm{Mn}^{2+}$-bound structure than to the 'locked' $\mathrm{Zn}^{2+}$-structure. This indicated that $\mathrm{Cd}^{2+}$ binding was a reversible process (Supplementary Table 5), consistent with the observation that $\mathrm{Cd}^{2}+$ could be extracted

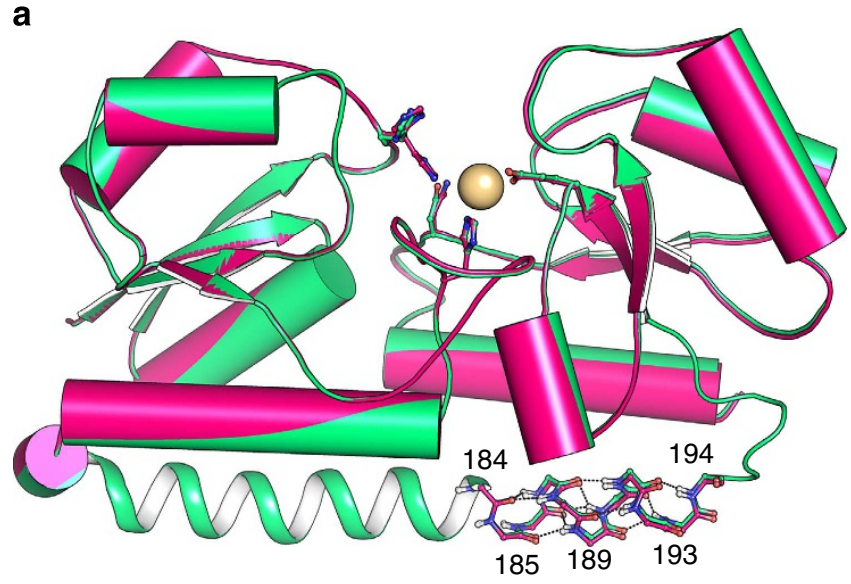

b

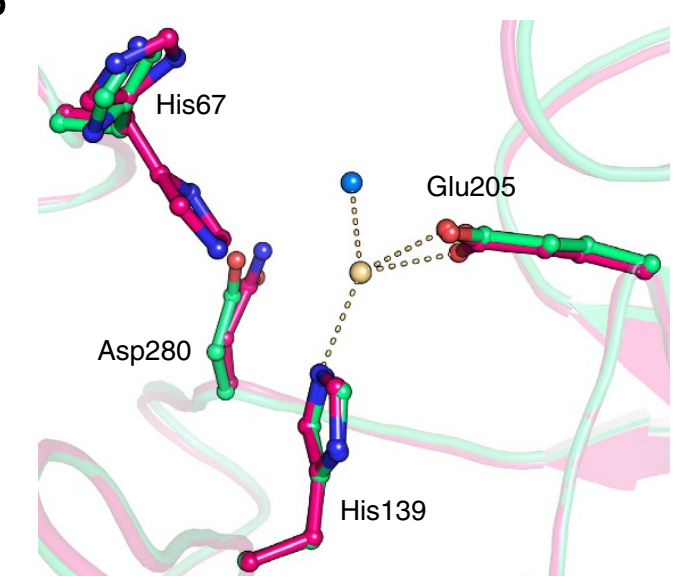

Figure 4 | Structure of $\mathbf{C d}^{2+}$-bound PsaA-D280N in an open conformation. (a) Superposition of $\mathrm{Cd}^{2}+$-bound PsaA-D28ON (magenta, PDB accession code 4UTO) and apo wild-type (green, PDB accession code 3ZK7) PsaA structures ( $C \alpha$ atoms, residues 32-163, were used for the superposition). The bound $\mathrm{Cd}^{2+}$ ion is shown as a pale-yellow sphere. The linking helix is shown in cartoon representation (up to residue 183) and backbone atoms in the flexible region of the linking helix (residues 184194) are shown in stick representation, with hydrogen bonds between backbone atoms as dashed lines. (b) Close-up view of the metal-binding sites between the superimposed $\mathrm{Cd}^{2+}$-bound PsaA-D280N (magenta, PDB accession code 4UTO) and apo wild-type (green, PDB accession code 3ZK7) PsaA structures. The bound $\mathrm{Cd}^{2+}$ ion is shown as a pale-yellow sphere and its coordination with the protein residues is shown as dashed lines. The water molecule coordinating the metal is shown as a light-blue sphere. In $\mathbf{a}, \mathbf{b}$, the metal-coordinating residues are shown in stick representation.

( $\sim 40 \%$ ) from the PsaA-Cd ${ }^{2+}$ complex by using the chelating agent EDTA (Fig. 3f). Thus, these findings provide further support for the conclusion that both $\mathrm{Mn}^{2+}$ and $\mathrm{Cd}^{2+}$ compete for transport via the Psa permease, consistent with the cellular accumulation data, which shows that $\mathrm{Mn}^{2+}$ uptake occurs at the expense of $\mathrm{Cd}^{2+}$ import and vice versa.

Reduced $\mathrm{Mn}^{2+}$ levels result in sensitivity to oxidative stress. We then sought to assess how $\mathrm{Cd}^{2}+$ affected oxidative stress management in S. pneumoniae. Manganese has a prominent role in regulating the expression of superoxide dismutase $(\operatorname{sod} A)$, where it also serves as a cofactor ${ }^{29-31}$. Transcription of $\operatorname{sodA}$ was decreased under $\mathrm{Cd}^{2+}$-induced $\mathrm{Mn}^{2+}$ starvation, but this was restored when supplemented with $\mathrm{Mn}^{2+}$ (Fig. 5a), and direct 
measurement of SodA activity from S. pneumoniae showed a similar trend (Fig. 5b). The impact of $\mathrm{Cd}^{2+}$ on oxidative stress response was ascertained using paraquat, which causes oxidative damage by promoting a futile redox cycle in the cytoplasm. We observed that although growth in the presence of $\mathrm{Cd}^{2}+$ resulted in a significant decrease in survival (Fig. 5c, $P=0.0087$ (twotailed unpaired $t$-test)), the increased sensitivity was not due to $\mathrm{Cd}^{2+}$, but instead was a result of the decreased $\mathrm{Mn}^{2+}$ accumulation, as $S$. pneumoniae grown in $30 \mu \mathrm{M} \mathrm{Mn}^{2+}: 30 \mu \mathrm{M}$ $\mathrm{Cd}^{2}+$ exhibited wild-type levels of survival (Fig. 5c). Hence, the perturbation of $\mathrm{Mn}^{2}+$ homeostasis by $\mathrm{Cd}^{2}+$ heightens the sensitivity to oxidative stress, while $\mathrm{Cd}^{2}+$ could be accumulated to high intracellular concentrations with no apparent deleterious effect. This ability to accumulate $\mathrm{Cd}^{2}+$ without direct toxicity indicated that intracellular buffering of the ion was crucial.

Reduced thiol groups on small peptides, primarily glutathione in S. pneumoniae, have been implicated in having an essential role in intracellular transition metal ion management $\left(\mathrm{Fe}^{2+}\right.$ and $\mathrm{Zn}^{2+}$ ) and in ameliorating $\mathrm{Cd}^{2+}$ toxicity ${ }^{32}$. S. pneumoniae is incapable of de novo glutathione synthesis and acquires glutathione via a high-affinity $\mathrm{ABC}$ permease, the Gsh permease $^{32}$. Here we investigated the contribution of glutathione to management of transition metal ion stress. In $S$. pneumoniae, the total glutathione content, in response to $\mathrm{Cd}^{2}+$ exposure, increased by approximately twofold to a mean ( \pm s.e.m.) pneumococcal cell concentration of $19.0 \pm 0.9 \mathrm{mM}$ (Fig. 5d). Glutathione abundance is crucial for $\mathrm{Cd}^{2+}$ management, as a mutant strain incapable of glutathione acquisition, S. pneumoniae $\Delta g s h T$, was hypersensitive to $\mathrm{Cd}^{2+}$ stress $\left(1 \mu \mathrm{M} \mathrm{Mn}^{2+}: 30 \mu \mathrm{M} \mathrm{Cd}^{2+}\right.$; Figs 2 and $\left.5 \mathrm{e}\right)$ and, in contrast to the wild type, could not be rescued by addition of $\mathrm{Mn}^{2+}$

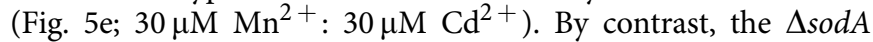
strain, which we have previously shown to be hypersensitive to oxidative stress challenge $e^{30,33}$, demonstrated a wild-type growth

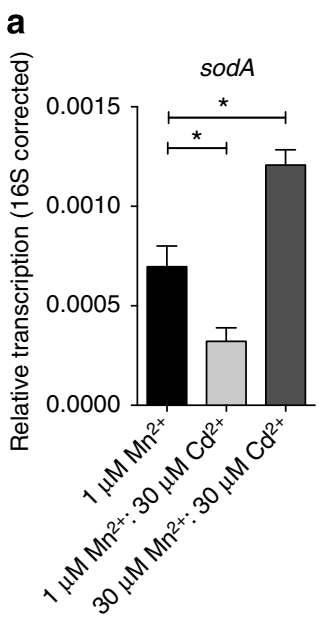

b

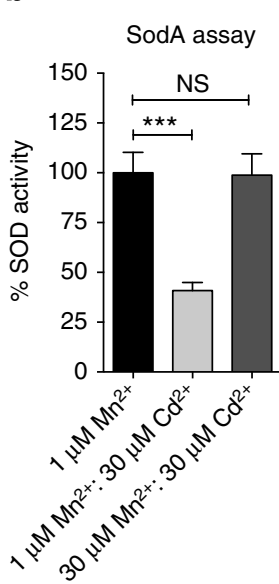

e

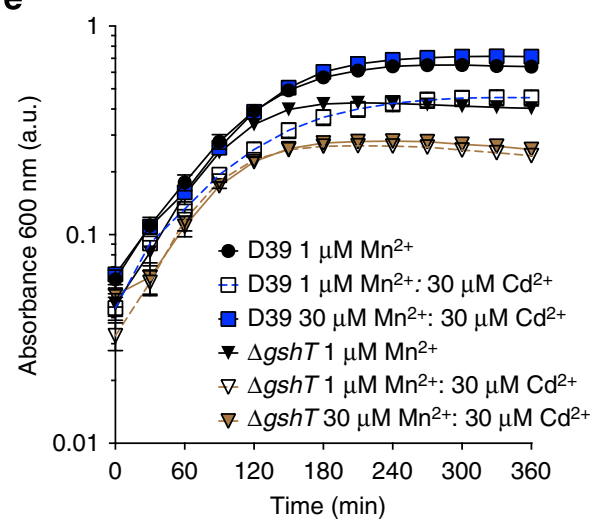

C
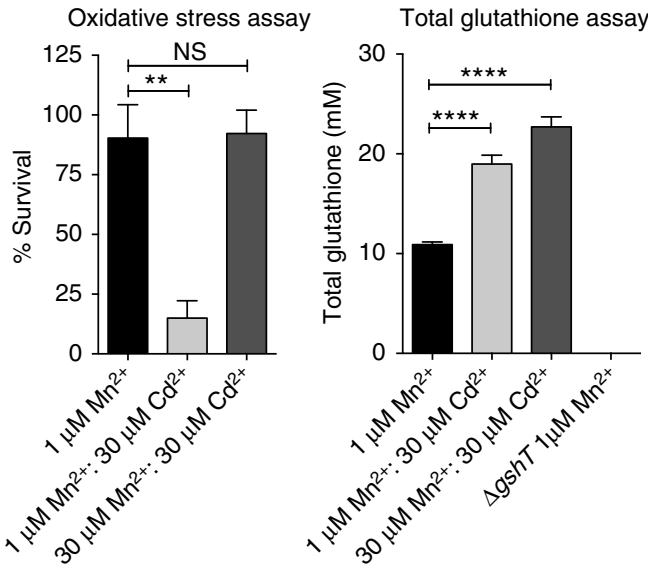

$\mathbf{f}$

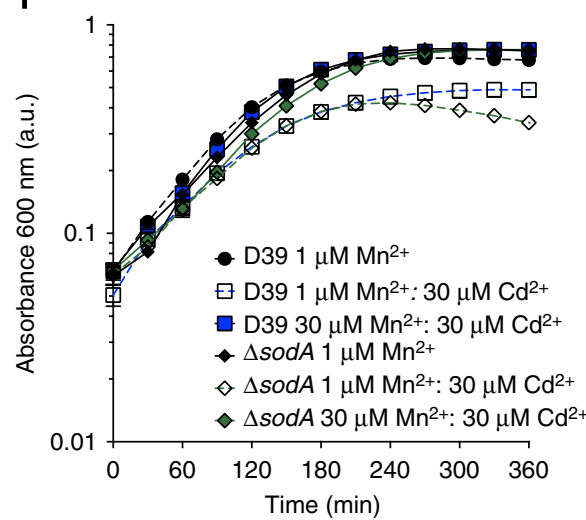

Figure 5 | The impact of $\mathbf{C d}^{2}+$ on sensitivity to oxidative stress. (a) Relative transcription, corrected to $16 \mathrm{~S}$ rRNA, of sodA by S. pneumoniae when

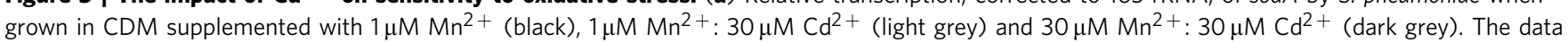
correspond to the mean ( \pm s.e.m.) of three independent biological experiments. (b) SodA activity assay S. pneumoniae when grown in CDM supplemented with $1 \mu \mathrm{M} \mathrm{Mn}^{2+}$ (black), $1 \mu \mathrm{M} \mathrm{Mn}^{2+}: 30 \mu \mathrm{M} \mathrm{Cd}^{2+}$ (light grey) and $30 \mu \mathrm{M} \mathrm{Mn}^{2+}: 30 \mu \mathrm{M} \mathrm{Cd}^{2+}$ (dark grey). Activity was calculated as a percentage of SodA activity observed for $S$. pneumoniae cells grown in $1 \mu \mathrm{M} \mathrm{Mn}^{2+}$. The data correspond to the mean ( \pm s.e.m.) of three independent biological experiments. (c) Paraquat killing of S. pneumoniae grown in CDM supplemented with $1 \mu \mathrm{M} \mathrm{Mn}^{2+}$ (black), $1 \mu \mathrm{M} \mathrm{Mn}{ }^{2+}: 30 \mu \mathrm{M} \mathrm{Cd} \mathrm{d}^{2+}$ (light grey) and $30 \mu \mathrm{M}$ $\mathrm{Mn}^{2+}: 30 \mu \mathrm{M} \mathrm{Cd}^{2+}$ (dark grey). Survival was calculated as a percentage of colonies at 30 min compared with cells not challenged with paraquat. The data correspond to the mean ( \pm s.e.m.) of three independent biological experiments. (d) Total glutathione determination per mean cell volume for S. pneumoniae grown in CDM supplemented with $1 \mu \mathrm{M} \mathrm{Mn}^{2+}$ (black), $1 \mu \mathrm{M} \mathrm{Mn}^{2+}: 30 \mu \mathrm{M} \mathrm{Cd}^{2+}$ (light grey) and $30 \mu \mathrm{M} \mathrm{Mn} \mathrm{M}^{2+}: 30 \mu \mathrm{M} \mathrm{Cd} d^{2+}($ dark grey). The statistical significance of the differences in the mean data for a-d was determined by two-tailed unpaired $t$-tests (NS, not significant; ${ }^{\star} P<0.05$, $\left.{ }^{\star \star} P<0.01,{ }^{\star \star \star} P<0.001,{ }^{\star \star \star \star} P<0.0001\right)$. (e,f) S. pneumoniae $\Delta g s h T(\mathbf{e})$ and $\Delta$ sodA (f) grown in CDM supplemented with metal ions as indicated. The data correspond to mean ( \pm s.e.m.) absorbance $600 \mathrm{~nm}$ measurements from three independent biological experiments. Errors bars, where not visible, are overlapped by the representative symbols. 
phenotype in the presence of $\mathrm{Cd}^{2+}$, with no apparent increase in sensitivity to metal ion stress (Figs 2 and 5f). Taken together, these findings indicate that glutathione serves a major role in $\mathrm{Cd}^{2}+$ buffering, whereas SodA appears to be dispensable, consistent with the lack of redox activity of $\mathrm{Cd}^{2}+$ ions.

To ascertain whether this was also the case for $\mathrm{Zn}^{2+}$, which is also presumed to be buffered by glutathione, we examined the response of the $\Delta g s h T$ strain to $\mathrm{Zn}^{2}+$ exposure. Intriguingly, while the $\Delta g s h T$ strain was insensitive to subinhibitory concentrations of $\mathrm{Zn}^{2+}$, similar to the $\Delta c z c D$ strain, the combination mutant $(\Delta g s h T / \Delta c z c D)$ showed significant attenuation of growth $\left(100 \mu \mathrm{M} \mathrm{Zn}{ }^{2+}\right.$; Fig. 2). At higher concentrations of $\mathrm{Zn}^{2+}$, the growth of the $\Delta g s h T / \Delta c z c D$ strain corresponded with the hypersensitive phenotype of the $\Delta c z c D$ strain, while the $\Delta g s h T$ strain showed a very mild impact, relative to wild type $(300 \mu \mathrm{M}$ $\mathrm{Zn}^{2+}$; Fig. 2). Taken together, these data show that intracellular management of $\mathrm{Zn}^{2}+$ occurs via the concerted actions of both glutathione and $\mathrm{Zn}^{2}+$ efflux, wherein the activation and efflux via $\mathrm{CzcD}$ is crucial to prevent $\mathrm{Zn}^{2+}$ intoxication. By contrast, management of $\mathrm{Cd}^{2+}$, which has no major efflux pathway, is predominantly dependent on cellular glutathione to prevent mismetallation of other proteins. Concordantly, when $\mathrm{Cd}^{2+}$ accumulation surpasses the buffering capacity of the glutathione

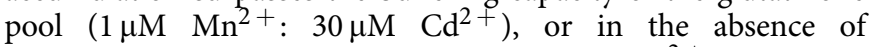
intracellular glutathione $(\Delta g s h T)$, this leads to $\mathrm{Cd}^{2}+$ toxicity.

\section{Discussion}

Anthropogenic-facilitated entry of non-physiological elements, such as $\mathrm{Cd}^{2+}$, into the biosphere presents unique cellular challenges for biological systems. The prior absence of these elements from the biosphere obviated any selective pressure towards evolving mechanisms for managing their impact on biological processes. As a consequence, many non-physiological elements rapidly accumulate in the food chain leading to significant toxicity in higher organisms ${ }^{1,2}$. We observed that $\mathrm{Cd}^{2+}$ was toxic to $S$. pneumoniae and that this arose from acute dysregulation of the transition metal ion homeostatic mechanisms. Elucidation of the total cellular quotient of metal ions in S. pneumoniae not only showed the impact of $\mathrm{Cd}^{2}+$ but also revealed novel aspects of cellular transition metal homeostasis. S. pneumoniae is a Gram-positive organism, comprising only a single cellular compartment; thus the concentration of transition metal ions accurately reflects their total intracellular accumulation and encompasses metal ions occurring in labile exchangeable pools and those bound weakly or tightly to proteins, peptides, nucleic acids and other molecules. Determination of the cellular quotient of metal ions in wild-type $S$. pneumoniae and mutant variants revealed the range over which cellular variation of transition metal ion concentrations is permissible. Manganese accumulation shows significant plasticity, with cells being viable at concentrations as low as $\sim 3 \%$ of typical accumulation levels ${ }^{23,30}$. By contrast, $\mathrm{Zn}^{2+}$ accumulation is regulated in a much narrower window, with its minimal cellular quotient being between 20 and $40 \%$ of typical accumulation. Intriguingly, the higher total cellular quotient of $\mathrm{Mn}^{2+}$ ions suggests that the concentration 'set-points' for cellular accumulation of transition metal ions may also contribute to ensuring efficient acquisition of metal cofactors by proteins. Manganese, which has been reported to be buffered by molecules such as citrate, phosphate and histidines residues from proteins in the cytoplasm ${ }^{34,35}$, has fewer reported cellular roles. Hence, its presence at such high cellular concentrations suggests that in S. pneumoniae it could occur predominantly as a labile pool of cytoplasmic-buffered $\mathrm{Mn}^{2+}$. By contrast $\mathrm{Zn}^{2+}$, which is reported in numerous studies to be highly utilized in intracellular proteins $^{36,37}$, has been predicted to have a significantly smaller labile pool ${ }^{10,36}$. Comprehensive intracellular metal ion speciation studies have been limited by a combination of technical and methodological challenges that remain to be surmounted. However, the data here allow us to speculate that, in S. pneumoniae, the potentially larger labile pool of $\mathrm{Mn}^{2+}$ ions, relative to $\mathrm{Zn}^{2}+$ ions, could be one mechanism that aids in $\mathrm{Mn}^{2+}$-dependent proteins correctly acquiring their cognate metal cofactor. The millimolar cellular quotient of $\mathrm{Zn}^{2+}$ ions in $S$. pneumoniae may also provide a plausible basis for the unexplained lower sensitivity of the zinc-responsive transcriptional regulator $\mathrm{AdcR}$ to $\mathrm{Zn}^{2+}$ ions, when compared with the homoplastic regulator, Zur, from E. coli ${ }^{10,15}$. In E. coli, $\mathrm{Zn}^{2}+$ has been shown to accumulate to an overall (cytoplasmic plus periplasmic) cellular concentration of $\sim 0.2 \mathrm{mM}$ and Zur is highly sensitive to $\mathrm{Zn}^{2}+$ abundance, sensing $\mathrm{Zn}^{2+}$ ions in the femtomolar range $\mathrm{e}^{10}$. By contrast, $\mathrm{AdcR}$ is $2-3$ orders of magnitude less sensitive, suggesting that a higher cellular 'setpoint' for sensing of $\mathrm{Zn}^{2+}$ ions in S. pneumoniae may exist ${ }^{15}$.

In the context of exposure to $\mathrm{Cd}^{2+}$, the impact on transition metal ion homeostasis corresponds to specific dysregulation of $\mathrm{Mn}^{2+}$ and $\mathrm{Zn}^{2+}$ accumulation, but via different mechanisms. Manganese accumulation was disrupted by direct competition of $\mathrm{Cd}^{2+}$ for the Psa permease and the upregulation of the $\mathrm{Mn}^{2+}$. efflux pathway MntE. These results also suggest that PsaR, the transcriptional regulator of the Psa permease, is not functionally activated by $\mathrm{Cd}^{2+}$ and thus permits upregulation of $p s a B C A$. In this way, the interaction of $\mathrm{Cd}^{2+}$ with PsaR may mimic the binding of $\mathrm{Zn}^{2+}$ rather than $\mathrm{Mn}^{2+}$, which occurs in a manner that does not permit repression of psaBCA transcription ${ }^{12}$. Although competition for the Psa permease was consistent with expectations, based on the inability of the SBP to be specific for solely $\mathrm{Mn}^{2}+$ ions, the mistranslocation of $\mathrm{Cd}^{2+}$ ions via the permease highlights an evolutionary limitation in managing extracellular stress by non-physiological elements. Specific acquisition of $\mathrm{Mn}^{2+}$ via the Psa permease arises from a combination of mechanisms that prevent the transport of $\mathrm{Zn}^{2}+$ ions, which are more abundant in the native niche of S. pneumoniae. However, the larger ionic radius and 'strained' coordination of the $\mathrm{Cd}^{2+}$ ion at the metal-binding site, similar to $\mathrm{Mn}^{2}+$ (Supplementary Table 3), resulted in an easily destabilized metal-protein complex, such that PsaA was incapable of preventing release of $\mathrm{Cd}^{2+}$ ions into the permease. This underscores the challenge encountered by metalloregulatory mechanisms when interacting with elements that have only recently entered the biosphere. In PsaA, the regulatory mechanisms that have evolved to prevent mistranslocation of $\mathrm{Zn}^{2+}$ ions are not capable of preventing the translocation of $\mathrm{Cd}^{2+}$ ions. By contrast with $\mathrm{Mn}^{2+}$, the $\mathrm{Cd}^{2+}$-induced disruption of $\mathrm{Zn}^{2}+$ homeostasis occurred via upregulation of $c z c D$, the $\mathrm{Zn}^{2+}$-efflux pathway, and the apparent inability of the pneumococcus to transcriptionally activate additional $\mathrm{Zn}^{2+}$. recruiting proteins (that is, AdcAII (ref. 13)) to compensate for the depletion of cellular $\mathrm{Zn}^{2+}$ ions. Hence, $\mathrm{Cd}^{2+}$ accumulation activated the coordinated pneumococcal physiological response to $\mathrm{Zn}^{2}+$ intoxication or overload ${ }^{13,15,17,23}$, resulting in the futile export and depletion of cellular $\mathrm{Zn}^{2}+$. Our findings suggest that $\mathrm{Cd}^{2}+$ accumulation results in the mismetallation of the $\mathrm{Zn}^{2+}$. responsive transcriptional regulators, AdcR and $\mathrm{SczA}^{13,15,17}$. Whether this is due to $\mathrm{Cd}^{2+}$ directly interacting with the $\mathrm{Zn}^{2+}$-binding sites of intracellular metal sensors ${ }^{38-40}$ or via $\mathrm{Cd}^{2+}$-mediated displacement of $\mathrm{Zn}^{2+}$ from thiol buffering sites $^{41}$, which results in the non-physiological activation of the $\mathrm{Zn}^{2+}$-responsive transcriptional regulators, remains to be determined. Irrespective of this fact, transcriptional activation of $c z c D$ is often construed as a mechanism to facilitate $\mathrm{Cd}^{2+}$ 
efflux based on studies of the archetypal protein from $C$. metallidurans ${ }^{18}$. However, in $S$. pneumoniae, similar to $S$. pyogenes $^{42}$, CzcD is primarily a $\mathrm{Zn}^{2+}$-efflux pathway and does not provide a major protective role against $\mathrm{Cd}^{2+}$ ions. As a consequence, in S. pneumoniae, the $\mathrm{Cd}^{2+}$-induced upregulation of $c z c D$ appears to preferentially deplete intracellular $\mathrm{Zn}^{2+}$, despite the greater abundance of $\mathrm{Cd}^{2}+$ ions.

$\mathrm{Cd}^{2+}$ accumulation is associated with oxidative stress, despite the redox-inert nature of the ion $^{4}$. The data here show that, in S. pneumoniae, the increased sensitivity to oxidative stress arises from the loss of $\operatorname{sodA}$ expression and activity due to $\mathrm{Cd}^{2+}$. induced $\mathrm{Mn}^{2+}$ depletion. However, the loss of SodA was an indirect by-product of $\mathrm{Cd}^{2+}$ accumulation and, importantly, not associated with the ability of the pneumococcus to tolerate $\mathrm{Cd}^{2+}$ ions. This was demonstrated by the $\Delta$ sodA strain, which showed wild-type growth phenotypes in the presence of extracellular $\mathrm{Cd}^{2+}$, and in wild-type $S$. pneumoniae, which retained the ability to accumulate high cellular concentrations of $\mathrm{Cd}^{2+}$ without an increase in sensitivity to oxidative stress, when supplemented with $\mathrm{Mn}^{2+}$. However, SodA still has a major role in the in vitro and in vivo fitness of S. pneumoniae owing to a lack of other mechanisms to detoxify superoxide ${ }^{23,30,33}$. Here, in the presence of $\mathrm{Cd}^{2+}$, the loss of SodA also occurs in conjunction with dysregulation of $\mathrm{Mn}^{2+}$ and $\mathrm{Zn}^{2+}$ homeostatic mechanisms and the accumulation of very high concentrations of $\mathrm{Cd}^{2+}$. Hence, it is the combination of these factors that compromises pneumococcal viability in the presence of $\mathrm{Cd}^{2+}$.

Intracellular accumulation of $\mathrm{Cd}^{2+}$ in $S$. pneumoniae was crucially dependent on glutathione, which acts as a low-affinity metal ion buffer for certain transition metal ions $\left(\mathrm{Fe}^{2+}, \mathrm{Zn}^{2+}\right.$ and $\left.\mathrm{Cd}^{2+}\right)$, as phenotypic growth perturbations were seen when $\mathrm{Cd}^{2+}$ accumulation exceeded the total cellular glutathione pool (19-23 mM). Although it cannot be completely discounted that glutathione contributes to pneumococcal oxidative stress management $^{32}$, the hypersensitivity of the $\Delta g s h T$ strain to extracellular $\mathrm{Cd}^{2+}$, in contrast to the $\Delta$ sodA strain, strongly implicates the major role for cellular glutathione is in metal ion buffering. The observed hypersensitivity of the $\Delta g s h T / \Delta c z c D$ strain to $\mathrm{Zn}^{2+}$ stress further underscores how intracellular metal ion homeostasis relies on the contributions of both cellular glutathione and the efflux machinery ${ }^{13,43}$. The results are also consistent with the central role of low-molecular-weight thiols for metal ion homeostasis in other microorganisms. Bacillithiol has recently been shown to have a crucial role in the buffering of $\mathrm{Zn}^{2+}$ ions in Bacillus subtilis ${ }^{41}$, while it also serves an undefined, but protective role in $\mathrm{Cd}^{2}+$ stress for $B$. subtilis and Staphylococcus aureus ${ }^{44}$. Although S. pneumoniae and other microorganisms lack a bacillithiol biosynthetic pathway, the role of low-molecular-weight species for metal ion homeostasis is emerging as a crucial mechanism. Overall, our findings here highlight the role of glutathione as an intracellular metal ion buffer, in preference to oxidative stress management. This finding has broad ramifications for the assumed role(s) of cellular glutathione, and related thiol derivatives, in prokaryotes and potentially eukaryotes ${ }^{43,41}$.

In conclusion, $\mathrm{Cd}^{2+}$ accumulation in the food chain and its toxicity are crucial, but largely overlooked concerns. Our findings reveal the molecular basis by which $\mathrm{Cd}^{2+}$ is mistranslocated via a $\mathrm{Mn}^{2+}$ transporter and provides new insights into how $\mathrm{Cd}^{2+}$ dysregulates intracellular transition metal homeostasis. Disruption of multiple essential metal ion homeostatic mechanisms is highly damaging to biological organisms, such as S. pneumoniae ${ }^{14}$, and in this way $\mathrm{Cd}^{2+}$ acts to severely dysregulate both $\mathrm{Mn}^{2+}$ and $\mathrm{Zn}^{2+}$ homeostasis (Supplementary Fig. 8). By revealing the remarkably high (millimolar) cellular quotients of transition metal ions and glutathione, this work also shows how $\mathrm{Cd}^{2+}$, despite its inability to directly generate reactive oxygen species, is associated with oxidative stress. This work provides a new understanding of the mechanisms by which $\mathrm{Cd}^{2+}$ enters cells and causes toxicity, and thereby, opens the way to identifying new routes towards developing specific therapeutic agents capable of preventing $\mathrm{Cd}^{2+}$ toxicity.

\section{Methods}

Growth experiments and whole-cell assays. The $S$. pneumoniae D39 $\Delta \operatorname{sod} A$, $\Delta c z c D, \Delta g s h T, \triangle m n t E, \triangle a d c C B A$ and $\triangle a d c A / \triangle a d c A I I$ strains have been generated previously ${ }^{13,32}$. The $\Delta m n t E$ and $\triangle g s h T / \Delta c z c D$ strains were generated as described previously ${ }^{13}$, using primer sequences listed in Supplementary Table 6. S. pneumoniae D39, $\Delta \operatorname{sod} A, \Delta c z c D, \Delta m n t E, \Delta g s h T, \Delta g s h T / \Delta c z c D, \Delta a d c C B A$ and $\triangle a d c A / \triangle a d c A I I$ were grown in CDM, which corresponded to the $\mathrm{C}+\mathrm{Y}$ media without transition metal ion supplementation ${ }^{23}$. The base ion content of the CDM was ascertained by ICP-MS on an Agilent $7500 \mathrm{cx}$ ICP-MS (Adelaide Microscopy, University of Adelaide $)^{23}$. Growth experiments were conducted in CDM

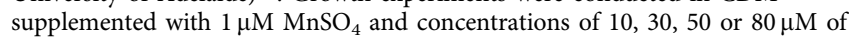
$\mathrm{CdCl}_{2}$ or $\mathrm{MnSO}_{4}$, as specified. All chemicals used in this study were purchased from Sigma-Aldrich, unless otherwise specified. For bacterial growth experiments, an inoculum was prepared from overnight grown culture on a blood-agar (BA) plate and resuspended in CDM to an absorbance at $600 \mathrm{~nm}\left(A_{600}\right)$ of 1.0 . The inoculum was then diluted in $200 \mu \mathrm{l}$ of CDM in a 96-well flat-bottom plate (Greiner Bio One) to a final $A_{600}$ of 0.05 and sealed with gas-permeable seal (Breathe-Easy, Diversified Biotech). The plate was then incubated in a FLUOStar Omega spectrophotometer with an ACU gas controller (BMG Labtech) at $310 \mathrm{~K}$ in $5 \% \mathrm{CO}_{2} . A_{600}$ readings were recorded using the well-scan function every $30 \mathrm{~min}$. Data from at least six independent growth experiments was averaged to ascertain the effect of $\mathrm{CdCl}_{2}$ on bacterial growth. Bacterial density at $A_{600}$ was measured as the c.f.u. per $\mathrm{ml}$ determined by serial dilutions on BA plates.

Bacterial growth for ICP-MS, paraquat killing and glutathione assays used identical growth parameters to the microplate experiments, with $\mathrm{MnSO}_{4}$ and $\mathrm{CdCl}_{2}$ supplementation as specified. For ICP-MS, $50 \mathrm{ml}$ of culture was grown to $A_{600}=0.3-0.4$, harvested and prepared for analysis by ICP-MS, as described previously $^{23}$. For the paraquat killing assays, $1 \mathrm{ml}$ of $A_{600}=0.3$ culture was incubated for $30 \mathrm{~min}$ with $10 \mathrm{mM}$ paraquat and then serially diluted and plated on blood agar. Total glutathione assays were performed using $1 \mathrm{ml}$ of $A_{600}=0.3$ culture with the Promega GSH-Glo assay kit according to the manufacturer's instructions (Promega, USA). To examine the effect of $\mathrm{Cd}^{2}+$ stress on cellular SodA activity, S. pneumoniae D39 was grown to an $A_{600}$ of 0.3 as for the ICP-MS experiments in CDM supplemented with $1 \mu \mathrm{M} \mathrm{MnSO}_{4}, 30 \mu \mathrm{M} \mathrm{CdCl}_{2}: 1 \mu \mathrm{M} \mathrm{MnSO}_{4}$ or $30 \mu \mathrm{M} \mathrm{CdCl}_{2}: 30 \mu \mathrm{M} \mathrm{MnSO}_{4}$. The cells were washed three times in PBS before lysis by sonication and intact cells and other insoluble material was removed by centrifugation at $277 \mathrm{~K}$ for $30 \mathrm{~min}$ at $14,000 \mathrm{~g}$. The assay was performed using a SOD activity kit (Calbiochem) and the data were normalized to the total protein concentration of the cell-free extract, as determined using the Dc Bio-Rad protein determination assay, followed by normalization (in percentage) to the average SOD activity measured for the untreated cells $(1 \mu \mathrm{M} \mathrm{MnSO} 4)$.

Metal sensitivity assays were also performed using a $S$. pneumoniae drop test. Bacteria were grown in CDM supplemented with $1 \mu \mathrm{M} \mathrm{MnSO}_{4}$ and grown until the $A_{600}$ reached $0.3-0.4$. Cells were then serially diluted 10 -fold up to a $10^{-5}$ dilution, and $5 \mu \mathrm{l}$ of each dilution were spotted on BA plates supplemented with varying concentrations of $\mathrm{ZnSO}_{4}(0,100,300$ and $1,000 \mu \mathrm{M})$ or $\mathrm{CdCl}_{2}(0,10,30$ and $100 \mu \mathrm{M})$. Plates were photographically documented following overnight incubation at $37^{\circ} \mathrm{C}, 5 \% \mathrm{CO}_{2}$.

RNA extraction and RT-PCR analysis. Pneumococci were grown as for the ICPMS analyses, then $500 \mu \mathrm{l}$ of the culture was mixed with $1 \mathrm{ml}$ of RNA Protect (Qiagen). RNA was extracted and purified using an RNeasy Protect Bacteria Mini Kit (Qiagen) after enzymatic lysis using lysozyme and mutanolysin, all according to the manufacturer's instructions. DNase I treatment (Roche) was performed followed by quantitative reverse transcription PCR using SuperScript III (Invitrogen) with a Roche LC480 Real-Time Cycler. The transcription levels of genes analysed were normalized to those obtained for $16 \mathrm{~S}$ rRNA. Primer sequences are in Supplementary Table 6

Cell volume and concentration determination. Scanning electron microscopy was used to determine the dimensions of the pneumococcal cell. Bacteria were grown, as described above, harvested and then prepared for, and analysed by a Philips XL30 FEG scanning electron microscope as described in ref. 45. Cell measurements were obtained using instrument software. The cell dimensions were used to calculate volume assuming an ellipsoid:

$$
V=\frac{4}{3} \pi r_{1} r_{2} r_{3}
$$

where $r_{1}, r_{2}$, and $r_{3}$ are the radii of the ellipsoidal cell in three dimensions determined by scanning electron microscopy (SEM). The total quotient of transition metal ions was then derived using cell volume ( $V$, litres), where the total 
cell density is known (c.f.u.), and concentration of metal ions in a sample of known volume and known number of cells $(M$, moles).

$$
C=\frac{(\mathrm{M} / \text { c.f.u. })}{V}
$$

The derived concentration represents mean molarity of metal ion per cell of mean dimensions.

Expression and purification of apo-PsaA. Recombinant PsaA was expressed in E. coli LEMO21(DE3) from the pCAMcLIC01-PsaA construct ${ }^{8}$. The dodecahistidine tag was removed from affinity-purified PsaA by enzymatic digestion by the human rhinovirus $3 \mathrm{C}$ protease and the protein purified further on a HisTrap HP column. Apo-PsaA was prepared by dialyzing the dodecahistidine tag-cleaved protein in a $20-\mathrm{kDa}$ molecular-weight-cutoff membrane (Slide-ALyzer, Pierce) against $4 \mathrm{l}$ of sodium acetate buffer, $\mathrm{pH} 4.0$, with $20 \mathrm{mM}$ EDTA at

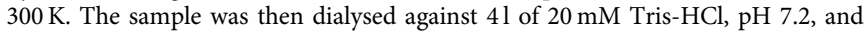
$100 \mathrm{mM} \mathrm{NaCl}$, at $277 \mathrm{~K}$ and centrifuged at $18,000 \mathrm{~g}$ for $10 \mathrm{~min}$ to remove any insoluble material. Protein samples were analysed for metal content by heating $5 \mu \mathrm{M}$ protein at $370 \mathrm{~K}$ for $15 \mathrm{~min}$ in $3.5 \% \mathrm{HNO}_{3}$ and the metal ion content was measured by ICP-MS

Protein assays. Protein concentration determination was performed using the Dc Bio-Rad protein determination assay. Metal-loading assays were performed on purified apo-PsaA $(30 \mu \mathrm{M})$ by mixing with $300 \mu \mathrm{M} \mathrm{CdCl}$ in a total volume of $2 \mathrm{ml}$ in the assay buffer ( $20 \mathrm{mM}$ MOPS, $\mathrm{pH} 7.2$, and $100 \mathrm{mM} \mathrm{NaCl}$ ) for $60 \mathrm{~min}$ at $277 \mathrm{~K}$. The sample was desalted on a PD10 column (GE Healthcare) into the assay buffer and the protein concentration was quantified. Protein was then either kept for ICP-MS analysis or mixed with $3 \mathrm{mM}$ EDTA in a total volume of $2 \mathrm{ml}$ for $60 \mathrm{~min}$ at $277 \mathrm{~K}$. Samples were then desalted on a PD10 column as before. Solutions (5$10 \mu \mathrm{M})$ of control, metal-loaded and EDTA-treated protein were prepared in $3.5 \%$ $\mathrm{HNO}_{3}$ and boiled for $15 \mathrm{~min}$ at $370 \mathrm{~K}$. Samples were then cooled and centrifuged for $20 \mathrm{~min}$ at $14,000 \mathrm{~g}$. The supernatant was then analysed by ICP-MS and the protein-to-metal ratio was determined.

Immunoblot analyses of PsaA expression levels. Wild-type and mutant $S$. pneumoniae were grown under the same conditions as for ICP-MS. After reaching an $A_{600}$ of 0.4 , cells were incubated with $0.1 \%$ sodium deoxycholate at $310 \mathrm{~K}$ for $60 \mathrm{~min}$ to induce lysis. Protein concentrations were determined and $10 \mu \mathrm{g}$ of total protein was loaded into each lane. After electrophoretic separation by SDS-PAGE, proteins were transferred to a nitrocellulose membrane using the iBlot (Life Technologies) system. The blots were incubated with murine anti-PsaA serum (1:2,000; ref. 23), followed by anti-mouse IRDye 800 (LI-COR; 1:50,000), and were scanned using an Odyssey infrared imaging system (LI-COR). Band intensities were measured using the manufacturer's application software and the results correspond to the mean ( \pm s.e.m.) of two independent biological experiments.

Determination of $\boldsymbol{K}_{\mathbf{D}}$ for PsaA with $\mathbf{C d}^{\mathbf{2}+}$. Excitation-emission spectra were determined on a FLUOStar Omega (BMG Labtech) at $301 \mathrm{~K}$ using black halfvolume 384-well microtitre plates (Greiner Bio One). All experiments were performed in $20 \mathrm{mM}$ MOPS (pH 6.7) and $50 \mathrm{mM} \mathrm{NaCl}$ with FluoZin-3 (Life Technologies) at a concentration of $50 \mathrm{nM}$. Deionized water and buffers were prepared and treated with Chelex-100 (Sigma-Aldrich) to avoid metal contamination. Filters used for FluoZin- 3 were excitation $(485 \pm 10 \mathrm{~nm})$ and emission $(520 \pm 5 \mathrm{~nm})$. To determine the dissociation constant between a metal (X) and FluoZin-3 (F), we considered the following equilibria:

$$
\mathrm{F}+\mathrm{X} \stackrel{K_{\mathrm{D} . \mathrm{X}}}{\leftrightarrow} \mathrm{F} . \mathrm{X}
$$

where, for a metal that increases the fluorescence of the probe by more than $\sim 10 \%$, the following equation, which is an exact analytical relationship derived from the mass action equation for the formation of a 1:1 complex between probe and metal, was used to estimate the dissociation constant, $K_{\mathrm{D} . \mathrm{x}}$

$$
\frac{f-f_{\min }}{f_{\max }-f}=\frac{[\mathrm{X}]}{K_{\mathrm{D} . \mathrm{X}}}
$$

where $f$ is the measured fluorescence intensity in the presence of metal, $f_{\max }$ is the fluorescence in the presence of saturating metal and $f_{\min }$ is the fluorescence in the absence of metal. In all cases, a low concentration $(50 \mathrm{nM})$ of probe was used and we assumed that the free-metal concentration was equal to the added metal concentration. The mean \pm s.e.m. $(n=6) K_{\mathrm{D}}$ determined for FluoZin-3 with $\mathrm{CdCl}_{2}$ in the buffer system used in this study was determined to be $250 \pm 35 \mathrm{nM}$. Competition by PsaA for $\mathrm{Cd}^{2+}$ binding was assessed by monitoring the decrease in the fluorescence of $50 \mathrm{nM}$ FluoZin-3-Cd ${ }^{2}+$ in response to increasing apo-PsaA concentrations and analysed using $\log _{10}$ [inhibitor] versus response model, with the experimentally derived $K_{\mathrm{D}}$ for FluoZin-3 with $\mathrm{Cd}^{2+}$, in GraphPad prism to determine the $K_{\mathrm{D}}$ value for $\mathrm{Cd}^{2}+$ binding by PsaA.
Protein crystallization and structure determination. Cd-bound PsaA-D280N protein crystals were obtained in $12.5 \%(\mathrm{w} / \mathrm{v})$ polyethylene glycol (PEG) 1000 , $12.5 \%$ (w/v) PEG 3350, 12.5\% (v/v) MPD, 0.1 M Trizma-Bicine, pH 8.7, and $0.01 \mathrm{M}$ $\mathrm{CdCl}_{2}$ using the hanging drop vapour diffusion method ${ }^{8} \cdot \mathrm{Cd}^{2+}$-bound wild-type PsaA crystals were grown in 26-36\% PEG 400, $0.1 \mathrm{M} \mathrm{NaCl}, 0.1 \mathrm{M}$ Trizma-HCl, pH 8.0 , and $0.1 \mathrm{M} \mathrm{CdCl}_{2}$ also using vapour diffusion. Before data collection, the crystals were flash-cooled by rapid immersion in liquid nitrogen. The diffraction data were collected on a single crystal at the Australian Synchrotron (MX2 beamline). $\mathrm{Cd}^{2+}$-bound D280N and wild-type PsaA structures were determined by molecular replacement with Phaser ${ }^{46}$ using the $\mathrm{Mn}^{2+}$-bound D280N (PDB accession code 3ZKA) and the $\mathrm{Zn}^{2+}$-bound (PDB accession code 1PSZ) PsaA crystal structures, respectively, as phasing models followed by automatic model building in Phenix.AutoBuild ${ }^{47}$. The structures were refined iteratively using Phenix.Refine and Refmac5 manual model fitting in $\operatorname{Coot}^{48}$. Both structures had two molecules (chains A and B) in each asymmetric unit and chain A molecules were used in subsequent structural analyses. Structural analyses (superpositions, metal ion coordination and $\mathrm{N}$-/C-terminal domain-crossing angles) were performed in Chimera ${ }^{49}$. Secondary structure elements were assigned by DSSP (version 2.1.0; ref. 50). The WHAT-IF ${ }^{51}$ and PISA $^{52}$ web services were used to identify hydrogen bonds and salt bridges. Data processing and structure refinement statistics can be found in Supplementary Table 7.

\section{References}

1. Nriagu, J. O. \& Pacyna, J. M. Quantitative assessment of worldwide contamination of air, water and soils by trace metals. Nature 333, 134-139 (1988).

2. Joseph, P. Mechanisms of cadmium carcinogenesis. Toxicol. Appl. Pharmacol. 238, 272-279 (2009).

3. Lane, T. W. et al. A cadmium enzyme from a marine diatom. Nature 435, 42 (2005).

4. Cuypers, A. et al. Cadmium stress: an oxidative challenge. Biometals 23, 927-940 (2010).

5. Archibald, F. S. \& Duong, M. N. Manganese acquisition by Lactobacillus plantarum. J. Bacteriol. 158, 1-8 (1984).

6. Hubert, N. \& Hentze, M. W. Previously uncharacterized isoforms of divalent metal transporter (DMT)-1: implications for regulation and cellular function. Proc. Natl Acad. Sci. USA 99, 12345-12350 (2002).

7. McAllister, L. J. et al. Molecular analysis of the psa permease complex of Streptococcus pneumoniae. Mol. Microbiol. 53, 889-901 (2004).

8. Couñago, R. M. et al. Imperfect coordination chemistry facilitates metal ion release in the Psa permease. Nat. Chem. Biol. 10, 35-41 (2014).

9. Dintilhac, A. \& Claverys, J. P. The adc locus, which affects competence for genetic transformation in Streptococcus pneumoniae, encodes an ABC transporter with a putative lipoprotein homologous to a family of streptococcal adhesins. Res. Microbiol. 148, 119-131 (1997).

10. Outten, C. E. \& O'Halloran, T. V. Femtomolar sensitivity of metalloregulatory proteins controlling zinc homeostasis. Science 292, 2488-2492 (2001).

11. Irving, H. \& Williams, R. J. P. The stability of transition-metal complexes. J. Chem. Soc. 3192-3210 (1953).

12. Lisher, J. P., Higgins, K. A., Maroney, M. J. \& Giedroc, D. P. Physical characterization of the manganese-sensing pneumococcal surface antigen repressor from Streptococcus pneumoniae. Biochemistry 52, 7689-7701 (2013).

13. Plumptre, C. D. et al. AdcA and AdcAII employ distinct zinc acquisition mechanisms and contribute additively to zinc homeostasis in Streptococcus pneumoniae. Mol. Microbiol. 91, 834-851 (2014).

14. Dintilhac, A., Alloing, G., Granadel, C. \& Claverys, J. P. Competence and virulence of Streptococcus pneumoniae: Adc and PsaA mutants exhibit a requirement for $\mathrm{Zn}$ and $\mathrm{Mn}$ resulting from inactivation of putative $\mathrm{ABC}$ metal permeases. Mol. Microbiol. 25, 727-739 (1997).

15. Reyes-Caballero, H. et al. The metalloregulatory zinc site in Streptococcus pneumoniae AdcR, a zinc-activated MarR family repressor. J. Mol. Biol. 403, 197-216 (2010).

16. Loisel, E. et al. AdcAII, a new pneumococcal Zn-binding protein homologous with $\mathrm{ABC}$ transporters: biochemical and structural analysis. J. Mol. Biol. 381, 594-606 (2008).

17. Kloosterman, T. G., van der Kooi-Pol, M. M., Bijlsma, J. J. \& Kuipers, O. P. The novel transcriptional regulator SczA mediates protection against $\mathrm{Zn}^{2}+$ stress by activation of the $\mathrm{Zn}^{2+}$-resistance gene $c z c D$ in Streptococcus pneumoniae. Mol. Microbiol. 65, 1049-1063 (2007).

18. Anton, A., Grosse, C., Reissmann, J., Pribyl, T. \& Nies, D. H. CzcD is a heavy metal ion transporter involved in regulation of heavy metal resistance in Ralstonia sp. strain CH34. J. Bacteriol. 181, 6876-6881 (1999).

19. Rosch, J. W., Gao, G., Ridout, G., Wang, Y. D. \& Tuomanen, E. I. Role of the manganese efflux system $m n t E$ for signalling and pathogenesis in Streptococcus pneumoniae. Mol. Microbiol. 72, 12-25 (2009).

20. Crupper, S. S., Worrell, V., Stewart, G. C. \& Iandolo, J. J. Cloning and expression of cadD, a new cadmium resistance gene of Staphylococcus aureus. J. Bacteriol. 181, 4071-4075 (1999).

21. Fu, Y. et al. A new structural paradigm in copper resistance in Streptococcus pneumoniae. Nat. Chem. Biol. 9, 177-183 (2013). 
22. Brenot, A., Weston, B. F. \& Caparon, M. G. A PerR-regulated metal transporter (PmtA) is an interface between oxidative stress and metal homeostasis in Streptococcus pyogenes. Mol. Microbiol. 63, 1185-1196 (2007).

23. McDevitt, C. A. et al. A molecular mechanism for bacterial susceptibility to zinc. PLoS Pathog. 7, e1002357 (2011).

24. Lawrence, M. C. et al. The crystal structure of pneumococcal surface antigen PsaA reveals a metal-binding site and a novel structure for a putative ABC-type binding protein. Structure 6, 1553-1561 (1998).

25. Braun, W. et al. Comparison of the NMR solution structure and the x-ray crystal structure of rat metallothionein-2. Proc. Natl Acad. Sci. USA 89, 10124-10128 (1992)

26. Graham, S. C., Maher, M. J., Simmons, W. H., Freeman, H. C. \& Guss, J. M Structure of Escherichia coli aminopeptidase P in complex with the inhibitor apstatin. Acta Crystallogr. D Biol. Crystallogr. 60, 1770-1779 (2004).

27. Price, N. M. \& Morel, F. M. M. Cadmium and cobalt substitution for zinc in a marine diatom. Nature 344, 658-660 (1990).

28. Xu, Y., Feng, L., Jeffrey, P. D., Shi, Y. \& Morel, F. M. Structure and metal exchange in the cadmium carbonic anhydrase of marine diatoms. Nature 452, 56-61 (2008).

29. Ogunniyi, A. D. et al. Central role of manganese in regulation of stress responses, physiology, and metabolism in Streptococcus pneumoniae. J. Bacteriol. 192, 4489-4497 (2010).

30. Eijkelkamp, B. A. et al. Extracellular zinc competitively inhibits manganese uptake and compromises oxidative stress management in Streptococcus pneumoniae. PLoS ONE 9, e89427 (2014).

31. Yesilkaya, H. et al. Role of manganese-containing superoxide dismutase in oxidative stress and virulence of Streptococcus pneumoniae. Infect. Immun. 68, 2819-2826 (2000)

32. Potter, A. J., Trappetti, C. \& Paton, J. C. Streptococcus pneumoniae uses glutathione to defend against oxidative stress and metal ion toxicity. J. Bacteriol. 194, 6248-6254 (2012).

33. Tseng, H. J., McEwan, A. G., Paton, J. C. \& Jennings, M. P. Virulence of Streptococcus pneumoniae: PsaA mutants are hypersensitive to oxidative stress. Infect. Immun. 70, 1635-1639 (2002).

34. Hider, R. C. \& Kong, X. L. Glutathione: a key component of the cytoplasmic labile iron pool. Biometals 24, 1179-1187 (2011).

35. Tabares, L. C. \& Un, S. In situ determination of manganese(II) speciation in Deinococcus radiodurans by high magnetic field EPR: detection of high levels of Mn(II) bound to proteins. J. Biol. Chem. 288, 5050-5055 (2013).

36. Sun, X. et al. Putative copper- and zinc-binding motifs in Streptococcus pneumoniae identified by immobilized metal affinity chromatography and mass spectrometry. Proteomics 11, 3288-3298 (2011).

37. Andreini, C., Bertini, I., Cavallaro, G., Holliday, G. L. \& Thornton, J. M. Metal ions in biological catalysis: from enzyme databases to general principles. J. Biol. Inorg. Chem. 13, 1205-1218 (2008).

38. Ralston, D. M. \& O'Halloran, T. V. Ultrasensitivity and heavy-metal selectivity of the allosterically modulated MerR transcription complex. Proc. Natl Acad. Sci. USA 87, 3846-3850 (1990).

39. Guerra, A. J. \& Giedroc, D. P. Metal site occupancy and allosteric switching in bacterial metal sensor proteins. Arch. Biochem. Biophys. 519, 210-222 (2012).

40. Sun, Y., Wong, M. D. \& Rosen, B. P. Role of cysteinyl residues in sensing Pb(II), $\mathrm{Cd}(\mathrm{II})$, and $\mathrm{Zn}(\mathrm{II})$ by the plasmid pI258 CadC repressor. J. Biol. Chem. 276, 14955-14960 (2001)

41. Ma, Z. et al. Bacillithiol is a major buffer of the labile zinc pool in Bacillus subtilis. Mol. Microbiol. 94, 756-770 (2014).

42. Ong, C. L., Gillen, C. M., Barnett, T. C., Walker, M. J. \& McEwan, A. G. An antimicrobial role for zinc in innate immune defense against group A Streptococcus. J. Infect. Dis. 209, 1500-1508 (2014).

43. Helbig, K., Bleuel, C., Krauss, G. J. \& Nies, D. H. Glutathione and transitionmetal homeostasis in Escherichia coli. J. Bacteriol. 190, 5431-5438 (2008).

44. Rajkarnikar, A. et al. Analysis of mutants disrupted in bacillithiol metabolism in Staphylococcus aureus. Biochem. Biophys. Res. Commun. 436, 128-133 (2013).
45. Fairbrother, L. et al. Effect of the cyanide-producing bacterium Chromobacterium violaceum on ultraflat Au surfaces. Chem. Geol. 265 , 313-320 (2009)

46. McCoy, A. J. Solving structures of protein complexes by molecular replacement with Phaser. Acta Crystallogr. D Biol. Crystallogr. 63, 32-41 (2007).

47. Terwilliger, T. C. et al. Iterative model building, structure refinement and density modification with the PHENIX AutoBuild wizard. Acta Crystallogr. D Biol. Crystallogr. 64, 61-69 (2008).

48. Emsley, P., Lohkamp, B., Scott, W. G. \& Cowtan, K. Features and development of Coot. Acta Crystallogr. D Biol. Crystallogr. 66, 486-501 (2010).

49. Pettersen, E. F. et al. UCSF Chimera--a visualization system for exploratory research and analysis. J. Comput. Chem. 25, 1605-1612 (2004).

50. Kabsch, W. \& Sander, C. Dictionary of protein secondary structure: pattern recognition of hydrogen-bonded and geometrical features. Biopolymers 22, 2577-2637 (1983).

51. Hekkelman, M. L. et al. WIWS: a protein structure bioinformatics Web service collection. Nucleic Acids Res. 38, W719-W723 (2010).

52. Krissinel, E. \& Henrick, K. Inference of macromolecular assemblies from crystalline state. J. Mol. Biol. 372, 774-797 (2007).

\section{Acknowledgements}

We acknowledge the use of the Australian Synchrotron (Victoria, Australia) and the University of Queensland Remote-Operation X-Ray (UQ-ROCX) Diffraction Facility. This work was supported by the Australian Research Council (ARC) grant DP120103957 to C.A.M. and J.C.P., and the National Health and Medical Research Council (NHMRC) Project grants 1022240, to C.A.M., and 1080784, to C.A.M. and M.J.M., and Program grants 565526, to A.G.M., B.K. and J.C.P., and 1071659, to B.K. and J.C.P. M.L.O. holds an ARC DECRA (DE1211550), B.K. is a NHMRC Senior Research Fellow (1003325) and J.C.P. is a NHMRC Senior Principal Research Fellow (1043070). We thank the beamline staff at the Australian Synchrotron for their support and Dr C. Adolphe for discussions.

\section{Author contributions}

S.L.B., B.A.E. and C.A.M. designed and executed all the biochemical studies. C.-l.Y.O. generated the mutant strains. Z.L., R.M.C. and M.J.M. designed and executed the crystallographic experiments. S.L.B., R.M.C., M.J.M., M.L.O. and C.A.M. drafted the manuscript. All authors contributed to the design, analysis, discussion of the research and writing of the final manuscript.

\section{Additional information}

Accession codes: Coordinates and structure factors for $\mathrm{Cd}^{2+}$-bound PsaA-D280N, partially occluded state, and for $\mathrm{Cd}^{2+}$-bound wild-type PsaA, closed state, have been deposited in the RCSB Protein Data Bank under accession codes 4uto and 4utp, respectively

Supplementary Information accompanies this paper at http://www.nature.com/ naturecommunications

Competing financial interests: The authors declare no competing financial interests

Reprints and permission information is available online at http://npg.nature.com/ reprintsandpermissions/

How to cite this article: Begg, S. L. et al. Dysregulation of transition metal ion homeostasis is the molecular basis for cadmium toxicity in Streptococcus pneumoniae. Nat. Commun. 6:6418 doi: 10.1038/ncomms7418 (2015).

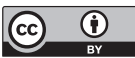

This work is licensed under a Creative Commons Attribution 4.0 International License. The images or other third party material in this article are included in the article's Creative Commons license, unless indicated otherwise in the credit line; if the material is not included under the Creative Commons license, users will need to obtain permission from the license holder to reproduce the material. To view a copy of this license, visit http://creativecommons.org/licenses/by/4.0/ 